\title{
A quasi-periodic minimal surface
}

\author{
Laurent Mazet and Martin Traizet
}

\begin{abstract}
We construct a properly embedded minimal surface in the flat product $\mathbb{R}^{2} \times \mathbb{S}^{1}$ which is quasi-periodic but not periodic.
\end{abstract}

Mathematics Subject Classification (2000). 53A10.

Keywords. Minimal surfaces, conjugate Plateau method, period problems, quasiperiodicity.

\section{Introduction}

Quasi-periodicity is a popular subject in both mathematics and physics. Probably the most famous examples are Penrose's quasi-periodic tiling, and quasi-periodic crystals.

Recall that a planar tiling $\mathcal{T}$ is quasi-periodic if any finite part of the tiling repeats infinitely many often. In other words, for arbitrary $R>0$, the tiling $\mathcal{T}$ contains an infinite number of translation copies of $\mathcal{T} \cap B(0, R)$ where $B(0, R)$ is the ball of radius $R$ centered at 0 .

Of course, for minimal surfaces, it is too much to ask that a part of the surface repeats exactly, because then by analytic continuation the whole surface would be periodic. We are thus led to the following definition, which was suggested to the authors by H. Rosenberg.

Definition 1. A complete minimal surface $M$ in $\mathbb{R}^{3}$ is quasi-periodic if there exists a diverging sequence of translations $\left(T_{n}\right)_{n \in \mathbb{N}}$, such that $T_{n}(M)$ converges smoothly to $M$ on compact subsets of $\mathbb{R}^{3}$.

While writing this paper, the authors discovered that the same notion had been introduced by Meeks, Perez and Ros in recent papers ([11], Theorem 1, [12], Definition 1, [13], Definition 1.5).

Of course a periodic minimal surface is quasi-periodic. A natural, and open, question is whether there exists quasi-periodic minimal surfaces which are not periodic. In this paper we answer this question when the ambient space is the flat product $\mathbb{R}^{2} \times \mathbb{S}^{1}$ instead of $\mathbb{R}^{3}$. The definition of quasi-periodicity is exactly the same in this case. 
Theorem 1. There exists a complete embedded minimal surface in $\mathbb{R}^{2} \times \mathbb{S}^{1}$ which is quasi-periodic but is not periodic. This surface has bounded curvature, infinite total curvature, infinite genus, infinitely many ends and two limit ends.

Let us now explain informally how this surface is constructed. H. Karcher has constructed a family of doubly periodic minimal surfaces in $\mathbb{R}^{3}$ which he called the "toroidal halfplane layers" [4]. They were the first complete, properly embedded, doubly periodic minimal surfaces to be found since H. Scherk's classical example. The toroidal halfplane layers have two periods: a horizontal period $T$ and a vertical period $(0,0,1)$. We may identify the quotient of $\mathbb{R}^{3}$ by the vertical period $(0,0,1)$ with $\mathbb{R}^{2} \times \mathbb{S}^{1}$. So the toroidal halfplane layers project to simply periodic minimal surfaces in $\mathbb{R}^{2} \times \mathbb{S}^{1}$, with period $T$. They have genus zero.

A very successful heuristic to construct new examples of minimal surfaces is to start from a simple example, and to complicate it by adding handles. One can start from a very symmetric example and break the symmetries by adding handles at suitable places.

Several people have added handles to H. Karcher's toroidal halfplane layers. The first one was F. Wei [18]. He was able to add one handle per fundamental piece in a
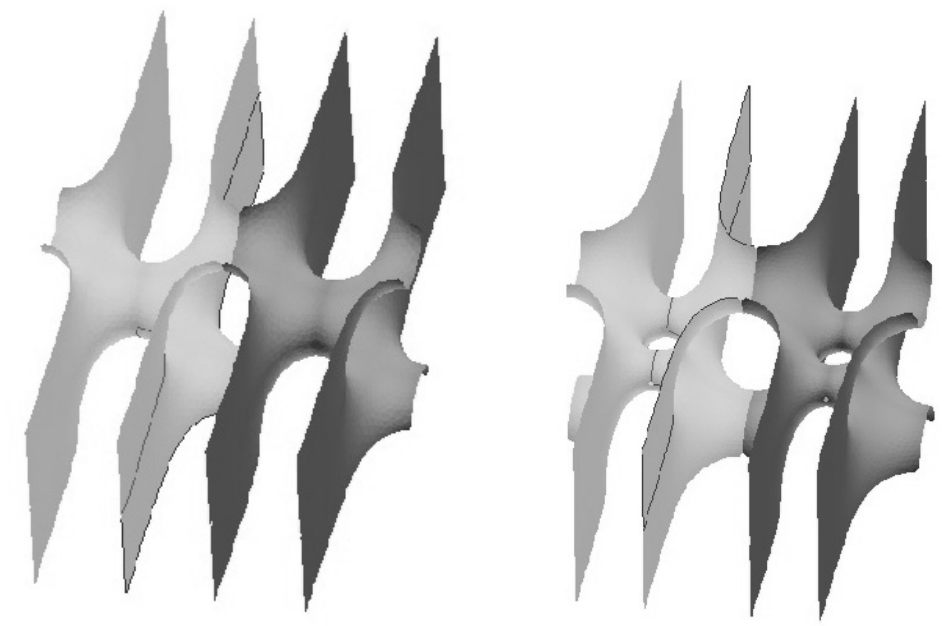

Figure 1. Left: one of Karcher's toroidal halfplane layers. Right: one of the Wei surfaces. A fundamental domain is highlighted for each. Both surfaces extend periodically vertically and horizontally. The fundamental domains of these two surfaces are the basic building blocks for the surface we construct: we assemble them in a quasi periodic, non periodic way. Computer images made by the authors using J. Hoffman's MESH software.

periodic way. The resulting surfaces have infinite genus in $\mathbb{R}^{2} \times \mathbb{S}^{1}$ and are periodic. W. Rossman, E. Thayer and M. Wolgemuth [16] have added handles in various ways 
to the toroidal halfplane layers, still requiring periodicity. Recently, the first author was able to add one single handle to the toroidal halfplane layers, without requiring horizontal periodicity. The resulting surfaces in $\mathbb{R}^{2} \times \mathbb{S}^{1}$ have genus one and are not periodic anymore.

In this paper we add an infinite number of handles to the toroidal halfplane layers, so the resulting surface have infinite genus, but without requiring horizontal periodicity. In fact the placement of the handles will be prescribed by a sequence of integers

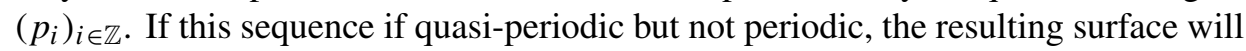
be quasi-periodic but not periodic.

To construct our surface, we follow the main lines of $\mathrm{H}$. Karcher's conjugate Plateau construction. The principle is to first construct a minimal surface with boundary made of straight edges. Typically this surface is constructed by solving a Dirichlet boundary value problem on a polygonal domain with piecewise linear boundary data. (The boundary data may take on infinite values, in which case this is called a JenkinsSerrin type problem.) Then one considers the conjugate minimal surface which is bounded by planar geodesics. If the polygonal boundary of the first surface is well chosen, the conjugate surface will extend by symmetry to an embedded, complete minimal surface. Adjusting the lengths of the edges so that this is the case is called the Period Problem. The difficulty of solving this problem is the main limitation of the method.

We add one more step to this construction. We first solve a Dirichlet boundary value problem for the maximal graph equation, with piecewise linear boundary data. Then we consider the conjugate function, whose graph is minimal. Then as above we consider the conjugate minimal surface, solve the Period Problem and extend by symmetry. So there are two consecutive conjugations, although of a different nature. The advantage of this approach is that part of the Period Problem (namely the vertical part) will be automatically solved. More details on maximal surfaces will be given in Section 2.

In our case, since we add infinitely many handles in a non-periodic way, we are faced with an infinite dimensional Period Problem. We begin by adding a finite number $N$ of handles. We solve the Period Problem using the Poincaré-Miranda Theorem, which is a natural $N$-dimensional extension of the intermediate value theorem. Then we let $N \rightarrow \infty$.

\section{Preliminaries}

2.1. Minimal and maximal graphs. Let $u$ be a function on a domain $\Omega \subset \mathbb{R}^{2}$. The graph of $u$ is a minimal surface if $u$ satisfies the minimal graph equation

$$
\operatorname{div}\left(\frac{\nabla u}{\sqrt{1+|\nabla u|^{2}}}\right)=0
$$


This equation is equivalent to the fact that the conjugate 1 -form

$$
d \Psi_{u}=\frac{u_{x}}{\sqrt{1+|\nabla u|^{2}}} d y-\frac{u_{y}}{\sqrt{1+|\nabla u|^{2}}} d x
$$

is closed. Locally, $d \Psi_{u}$ is then the differential of a function $\Psi_{u}$ called the conjugate function. Then $v=\Psi_{u}$ is a solution of the maximal graph equation

$$
\operatorname{div}\left(\frac{\nabla v}{\sqrt{1-|\nabla v|^{2}}}\right)=0
$$

This is called the maximal graph equation because $v$ satisfies (2) if its graph is a maximal surface in the Lorentzian space $\mathbb{L}^{3}$, namely a space-like surface which is a critical point for the area functional.

Conversely, let $v$ be a solution of (2). Then the conjugate 1-form

$$
d \Phi_{v}=\frac{v_{y}}{\sqrt{1-|\nabla v|^{2}}} d x-\frac{v_{x}}{\sqrt{1-|\nabla v|^{2}}} d y
$$

is closed. Hence locally $d \Phi_{v}$ is the differential of a function $u=\Phi_{v}$ which solves the minimal graph equation (1). Moreover up to a constant, $\Phi_{\Psi_{u}}=u$.

2.2. The Dirichlet boundary value problem. Let $\Omega \subset \mathbb{R}^{2}$ be a bounded domain. Let $v: \Omega \rightarrow \mathbb{R}$ be a smooth function satisfying (2). Then $|\nabla v|<1$ hence $v$ is Lipschitz and extends continuously to $\partial \Omega$, so we can talk about the boundary values of $v$. (For this to be true, we need some regularity of the boundary of $\Omega$. All the domains we consider will have piecewise smooth boundary.)

We need to construct solutions $v$ of the maximal graph equation (2) in $\Omega$, with prescribed boundary values, and with singularities at some prescribed points inside $\Omega$. For this we use the following theorem, which is a consequence of Theorem 1 in [5] and Theorem 4.1 in [2]:

Theorem 2. Let $\Omega \subset \mathbb{R}^{2}$ be a bounded domain. Let $s \subset \Omega$ be a finite set (the singular set). Let $\varphi: \partial \Omega \cup \& \rightarrow \mathbb{R}$ be a given function such that

$$
\left|\varphi(p)-\varphi\left(p^{\prime}\right)\right| \leq d_{\Omega}\left(p, p^{\prime}\right) \text { for all } p, p^{\prime} \in \partial \Omega \cup \S, p \neq p^{\prime},
$$

where the inequality is strict whenever the segment $\left[p, p^{\prime}\right]$ is not contained in $\partial \Omega$. Then there exists a function $v: \Omega \rightarrow \mathbb{R}$ which satisfies the maximal graph equation (2) in $\Omega \backslash 8$, with boundary data $v=\varphi$ on $\partial \Omega \cup 8$. This function is smooth in $\Omega \backslash 8$. (Here $d_{\Omega}$ is the intrinsic distance of $\Omega$, so if $\Omega$ is convex, it agrees with the Euclidean distance.) 
2.3. Some complements on the correspondence $v \leftrightarrow \Phi_{v}$. Since we obtain solutions $v$ to the Dirichlet problem for (2), we need to understand the behaviour of the conjugate function $\Phi_{v}$ near the boundary. The first result describes the behaviour near the boundary of the domain $\Omega$.

Lemma 1 ([3] and [9]). Let $v$ be a solution of (2) on $\Omega$ and $T \subset \partial \Omega$ be an open straight segment oriented as $\partial \Omega$. Then $\int_{T} d v=|T|$ if and only if $\Phi_{v}$ diverges to $+\infty$ on $T$.

Now we shall describe the behaviour near a singularity in the domain. Let $v$ be a solution of (2) on a punctured disk $\mathbb{D}^{*}$ (with $\mathbb{D}=\left\{(x, y) \in \mathbb{R}^{2} \mid x^{2}+y^{2}<r^{2}\right\}$ ). Then the conjugate function $u=\Phi_{v}$ is not well defined on $\mathbb{D}^{*}$; actually, $u$ is multivalued in the sense that when we turn around the origin we need to add a constant to $u$ : this constant is given by $\int_{\gamma} d \Phi_{v}$ where $\gamma$ generates $\pi_{1}\left(\mathbb{D}^{*}\right)$. If this constant vanishes, $u$ is well defined and so extends smoothly to the whole disk; $v$ then also extends to $\mathbb{D}$ and the origin is a removable singularity for $v$.

In the case $\int_{\gamma} d \Phi_{v} \neq 0$, the graph of the multivalued function $u$ has then the shape of a half-helicoid. On the boundary of the cylinder $\mathbb{D} \times \mathbb{R}$ the graph is bounded by a helix-like looking curve. It is bounded by a vertical straight line above the origin.

In fact in the paper, we are always in the case where $v$ is positive and vanishes at the origin. This first implies that $\int_{\gamma} d \Phi_{v} \neq 0$. Besides, Theorem 4.2 in [9] proves that the graph of $u$ is bounded by a vertical straight line above the origin.

2.4. Convergence of sequences of solutions. We shall study many times the convergence or the divergence of sequences of solutions to (2). In this subsection, we expose some results that we will use. Actually, these results were developed by the first author in [7], [9] for solutions of (1); the correspondence $u \leftrightarrow \Psi_{u}$ and $v \leftrightarrow \Phi_{v}$ translates them to solutions of (2). Here the convergence that we shall consider is the $C^{k}$ convergence on compact subsets of the domain for every $k$.

So let us consider a sequence $\left(v_{n}\right)_{n \in \mathbb{N}}$ of solutions to (2) which are defined on a domain $\Omega$. We first notice that since each $v_{n}$ is Lipschitz continuous there exists a subsequence of $\left(v_{n}-v_{n}(q)\right)$ (where $q \in \Omega$ is a fixed point) that converges to a Lipschitz function $v$ on $\Omega$; but the convergence is only $C^{0}$ and $v$ is a priori not a solution to (2). However, since we have convergence on $\bar{\Omega}$, we can talk about the boundary value of the limit.

To study the smooth convergence, we first define the convergence domain of the sequence by

$$
\mathscr{B}\left(\left(v_{n}\right)_{n \in \mathbb{N}}\right)=\left\{p \in \Omega \mid \sup _{n}\left\{\left|\nabla v_{n}\right|(p)\right\}<1\right\} .
$$

$\mathscr{B}\left(\left(v_{n}\right)_{n \in \mathbb{N}}\right)$ is an open subset of $\Omega$ and on each component $\Omega^{\prime}$ of it, there is a subsequence of $\left(v_{n}-v_{n}(q)\right)_{n \in \mathbb{N}}$ converging $C^{k}$ on compact subsets of $\Omega^{\prime}$ to a 
solution $v$ of (2), where $q$ is some fixed point in $\Omega^{\prime}$. We notice that all solutions of (2) that we shall consider are bounded by 1 ; thus we do not need to use the vertical translation by $v_{n}(q)$ to ensure the convergence. Besides $\Omega \backslash \mathscr{B}\left(\left(v_{n}\right)_{n \in \mathbb{N}}\right)$ is the union of straight lines $\cup_{i} L_{i}$, where each $L_{i}$ is a component of the intersection of a straight line with $\Omega$. The $L_{i}$ are called divergence lines of the sequence $\left(v_{n}\right)_{n \in \mathbb{N}}$ since $\sup _{n}\left\{\left|\nabla v_{n}\right|(p)\right\}=1$ for $p \in L_{i}$; more precisely we have

Lemma 2. Let p be a point in a divergence line L, then a subsequence of $\left(\nabla v_{n}(p)\right)_{n \in \mathbb{N}}$ converges to one of the two unit generating vectors of $L$.

Besides, if $T$ is a segment in $L$, it holds that $\int_{T} d v_{n} \rightarrow \pm|T|$ for a subsequence.

To ensure the convergence of a subsequence of $\left(v_{n}\right)_{n \in \mathbb{N}}$ on $\Omega$ it then suffices to prove there are no divergence line. The above lemma is one tool in that direction. The following one is another.

Lemma 3. Let us assume that one part of the boundary of $\Omega$ is a segment $[a, b]$. If for every $n\left|v_{n}(a)-v_{n}(b)\right|=|a-b|$, then no divergence line can end in the interior of $[a, b]$.

Actually in this paper, the solutions are not defined on the same domain $\Omega$ : we have in fact a sequence of domains $\left(\Omega_{n}\right)_{n \in \mathbb{N}}$ and each solution $v_{n}$ is defined on $\Omega_{n}$. So to make sense to the above definition we need to introduce the limit domain $\Omega_{\infty}$ :

$$
\Omega_{\infty}=\bigcup_{p \in \mathbb{N}} \operatorname{Int}\left(\bigcap_{k \geq p} \Omega_{k}\right)
$$

A point is then in $\Omega_{\infty}$ if a neighborhood of this point is included in all $\Omega_{k}$ for $k$ great enough. With this definition, we have anew the convergence domain and the divergence lines by replacing $\Omega$ by $\Omega_{\infty}$.

We notice that when $\left(\Omega_{n}\right)_{n \in \mathbb{N}}$ is an increasing sequence, $\Omega_{\infty}$ is simply the union of all the $\Omega_{n}$. In this paper, the sequence $\Omega_{n}$ is often $\Omega \backslash \delta_{n}$ where $s_{n}$ is a locally finite set of points. If $\left(\delta_{n}\right)$ converges on compact subsets to a locally finite subset $\delta_{\infty}$ then $\Omega_{\infty}=\Omega \backslash \wp_{\infty}$.

\section{The fundamental piece}

3.1. The Dirichlet boundary value problem. In this section we solve a Dirichlet boundary value problem for the maximal graph equation (2) in an infinite strip. The solution $v$ will have singularities at some prescribed points. The position of these singularities are the parameters of our construction. (Each singularity is responsible for one handle of the minimal surface we are constructing. In later sections, we will adjust these parameters so that the Period Problem is solved.) 
Fix some $\ell>0$ and let $\Omega$ be the strip $\mathbb{R} \times(-\ell, \ell)$. Let us define the boundary data $\varphi$ on $\partial \Omega$ as follows: for $k \in \mathbb{Z}$, let $a_{k}^{+}=(k, \ell)$ and $a_{k}^{-}=(k,-\ell)$. Define $\varphi$ on the segment $\left[a_{2 k-1}^{ \pm}, a_{2 k+1}^{ \pm}\right]$by $\varphi(p)=\left|p-a_{2 k}^{ \pm}\right|$. In other words $\varphi$ is piecewise affine on $\partial \Omega$, with value 0 at $a_{2 k}^{ \pm}$and 1 at $a_{2 k+1}^{ \pm}$(see Figure 2).

Let $\delta$ be a closed, discrete subset of the horizontal line $y=0$. It will be convenient to identify the $x$-axis with $\mathbb{R}$ and see $\delta$ as a subset of $\mathbb{R}$. When $\delta$ is finite, we write $\delta=\left\{q_{1}, \ldots, q_{N}\right\}$ and assume that $q_{1}<q_{2}<\cdots<q_{N}$. When $\delta$ is infinite, we may write $\delta=\left\{q_{i}: i \in I\right\}$, with $q_{i}<q_{i+1}$, where $I$ is either $\mathbb{N},-\mathbb{N}$ or $\mathbb{Z}$, depending on whether $\delta$ is bounded from below, bounded from above, or neither. Finally, we define $\varphi=0$ on $\&$.

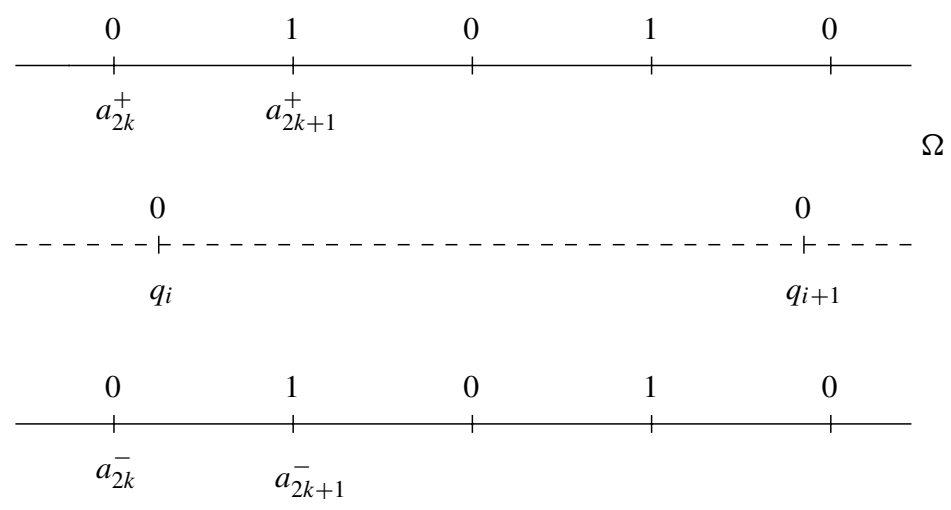

Figure 2. The Dirichlet boundary value problem.

Proposition 1. Let $\Omega$ and $\&$ be as above. Assume that

$$
\left|q-a_{2 k+1}^{+}\right|>1 \text { for all } q \in \delta, k \in \mathbb{Z} .
$$

Then there exists a function $v$ on $\Omega$ which solves (2) in $\Omega \backslash 8$, with boundary data $v=\varphi$ on $\partial \Omega \cup$ \&. Moreover, $0 \leq v \leq 1$ in $\Omega$. The function $v$ is unique.

We call the function $v$ the solution to the Dirichlet problem in $\Omega \backslash \&$. When needed, we will write $v=v[8]$.

Remark 1. The condition (4) is automatically satisfied when $\ell>1$. We are however mostly interested in the case $\ell<1$, as this is the only case where we know how to solve the Period Problem.

Proof of the proposition. For $n \in \mathbb{N}^{*}$, consider the box $\Omega_{n}=(-2 n, 2 n) \times(-\ell, \ell)$. We first solve the Dirichlet problem on $\Omega_{n}$ and then let $n \rightarrow \infty$. Let $\delta_{n}=\varsigma \cap \Omega_{n}$. 
Define $\varphi_{n}$ on $\partial \Omega_{n} \cup \wp_{n}$ by $\varphi_{n}=\varphi$ on the horizontal edges $[-2 n, 2 n] \times\{ \pm \ell\}, \varphi_{n}=0$ on the vertical edges $\{ \pm 2 n\} \times[-\ell, \ell]$, and $\varphi_{n}=0$ on the singular set $s_{n}$.

Claim 1. The function $\varphi_{n}$ on $\partial \Omega_{n} \cup \wp_{n}$ satisfies the condition (3) of Theorem 2.

Proof. Consider $p, p^{\prime} \in \partial \Omega_{n} \cup s_{n}, p \neq p^{\prime}$.

- If $p$ and $p^{\prime}$ are both on the line $y=\ell$, then clearly $\left|\varphi_{n}(p)-\varphi_{n}\left(p^{\prime}\right)\right| \leq\left|p-p^{\prime}\right|$.

- If $p$ and $p^{\prime}$ are on $\partial \Omega_{n}$, let $\tilde{p}$ and $\tilde{p}^{\prime}$ be the projections of $p$ and $p^{\prime}$ on the line $y=\ell$. Then

$$
\left|\varphi_{n}(p)-\varphi_{n}\left(p^{\prime}\right)\right|=\left|\varphi_{n}(\tilde{p})-\varphi_{n}\left(\tilde{p}^{\prime}\right)\right| \leq\left|\tilde{p}-\tilde{p}^{\prime}\right| \leq\left|p-p^{\prime}\right| .
$$

Moreover, if the segment $\left[p, p^{\prime}\right]$ is not horizontal, the last inequality is strict. If the segment $\left[p, p^{\prime}\right]$ is horizontal, and is not included in $\partial \Omega_{n}$, then $p$ and $p^{\prime}$ are both on the vertical edges, so $\left|\varphi_{n}(p)-\varphi_{n}\left(p^{\prime}\right)\right|=0<\left|p-p^{\prime}\right|$ as required.

- If $p$ is on $\partial \Omega_{n}$ and $p^{\prime}=q \in s$ : if $p$ is on a vertical edge, then $\varphi_{n}(p)=\varphi_{n}(q)=$ 0 . If $p$ is on the segment $\left[a_{2 k}^{+}, a_{2 k+1}^{+}\right]$, we have

$$
\left|\varphi_{n}(p)-\varphi_{n}(q)\right|=\left|p-a_{2 k}^{+}\right|=1-\left|p-a_{2 k+1}^{+}\right| \leq 1+|q-p|-\left|q-a_{2 k+1}^{+}\right|<|p-q|
$$

where we have used the triangle inequality and the hypothesis of Proposition 1. The case where $p$ is on the segment $\left[a_{2 k-1}^{+}, a_{2 k}^{+}\right]$is similar, and the case where $p$ is on the line $y=-\ell$ follows by symmetry of $\varphi_{n}$.

- If $p, p^{\prime}$ are both in $\&$, then $\varphi_{n}(p)=\varphi_{n}\left(p^{\prime}\right)=0$.

By Theorem 2, there exists a solution $v_{n}$ of the maximal graph equation (2) on $\Omega_{n} \backslash \delta_{n}$ with boundary data $\varphi_{n}$. Since $v_{n}$ extends continuously to the compact set $\bar{\Omega}_{n}, v_{n}$ is bounded. By the maximum principle for the maximal graph equation, $v_{n}$ reaches its maximum and its minimum at a boundary point or a singular point, so $0 \leq v_{n} \leq 1$ in $\Omega_{n}$. Consider now the sequence $\left(v_{n}\right)_{n}$. Let $L$ be a divergence line. Let $T \subset L$ be a segment, then $\lim \int_{T} d v_{n}= \pm|T|$. Since $v_{n}$ is bounded, this implies that $L$ has finite length so $L$ is a segment connecting two points $p$ and $p^{\prime}$ on $\partial \Omega \cup \S$. Then

$$
\left|\varphi(p)-\varphi\left(p^{\prime}\right)\right|=\left|\int_{p}^{p^{\prime}} d v_{n}\right| \rightarrow\left|p-p^{\prime}\right| \Rightarrow\left|\varphi(p)-\varphi\left(p^{\prime}\right)\right|=\left|p-p^{\prime}\right|
$$

which contradicts claim 1 since $L \subset \Omega$. Hence there are no divergence lines, so passing to a subsequence, $\left(v_{n}\right)_{n}$ converges on compact subsets of $\bar{\Omega}$ to a function $v$, which is a solution of (2) in $\Omega \backslash \&$ with boundary data $\varphi$ on $\partial \Omega \cup \&$. Uniqueness follows from Theorem 2 in [10]. 
3.2. The minimal graph. In this subsection and the following one, we assume that $\delta=\left\{q_{1}, \ldots, q_{N}\right\}$ with $q_{1}<\cdots<q_{N}$. Let $v$ be the solution of the Dirichlet problem on $\Omega \backslash \&$, given by Proposition 1 . Let $\Omega^{+}$be the strip $\mathbb{R} \times(0, \ell)$. Since $\Omega^{+}$is simply connected and $v$ is smooth in $\Omega^{+}$, the conjugate function $u$ is well defined (up to a constant) in $\Omega^{+}$. The graph of $u$ is a minimal surface. In this section we describe geometrically its boundary.

By uniqueness, $v$ satisfies $v(x,-y)=v(x, y)$ in $\Omega$. Hence on the $x$-axis, away from the singular points $q_{1}, \ldots, q_{N}$, we have $v_{y}=0$. From the definition of $\Phi_{v}$, this gives $u_{x}=0$. Hence $u$ is locally constant on the $x$-axis minus the singular points, with a finite number of jumps at the points $q_{1}, \ldots, q_{N}$. On the line $y=\ell, u$ goes to $+\infty$ on the segments $\left(a_{2 k-1}^{+}, a_{2 k}^{+}\right)$and to $-\infty$ on the segments $\left(a_{2 k}^{+}, a_{2 k+1}^{+}\right), k \in \mathbb{Z}$ (see Lemma 1).

Let $M$ be the graph of $u$ on the strip $\Omega^{+}$. The minimal surface $M$ is bounded by vertical lines $A_{k}$ above the points $a_{k}^{+}, k \in \mathbb{Z}$, by $N$ vertical segments $B_{i}$ above the points $q_{i}, i=1, \ldots, N$, by $N-1$ horizontal segments $C_{i}$ above the segments $\left(q_{i}, q_{i+1}\right)$, $i=1, \ldots, N-1$ and by two horizontal half-lines $C_{0}$ and $C_{N}$ above $\left(-\infty, q_{1}\right)$ and $\left(q_{N},+\infty\right)$ (see Figure 3). The heights of the horizontal pieces $C_{0}, \ldots, C_{N}$ are unknown.

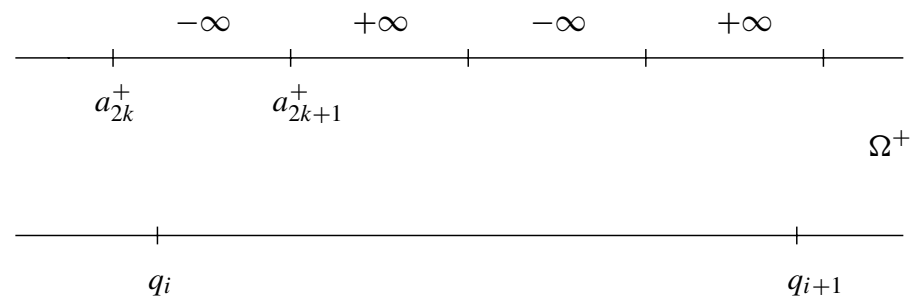

Figure 3. The minimal graph.

3.3. The conjugate minimal surface. Let $M^{*}$ be the conjugate minimal surface to $M$. The third coordinate of $M^{*}$ (seen as an immersion of the strip $\Omega^{+}$) is the function $v$, so $M^{*}$ lies in the slab $0 \leq z \leq 1$. Let $A_{k}^{*}, B_{i}^{*}$ and $C_{i}^{*}$ denote the corresponding conjugate curves on $M^{*}$ (see Figure 4). Then the $A_{k}^{*}, k \in \mathbb{Z}$, and $B_{i}^{*}$, $i=1, \ldots, N$, are horizontal geodesics. From the boundary values of $v, A_{2 k}^{*}$ and $B_{i}^{*}$ lie in the plane $z=0$, while $A_{2 k+1}^{*}$ lies in the plane $z=1$. Each $C_{i}^{*}, i=0, \ldots, N$ is a geodesic contained in a vertical plane parallel to the plane $x=0$. There is no reason however that all $C_{i}^{*}$ are in the same vertical plane: this is the Period Problem, which we will consider in the next section.

Remark 2. The function $u$ is the solution of a Jenkins-Serrin type problem on the strip $\Omega^{+}$. One possible way to construct $M$ would be to directly solve this Jenkins- 


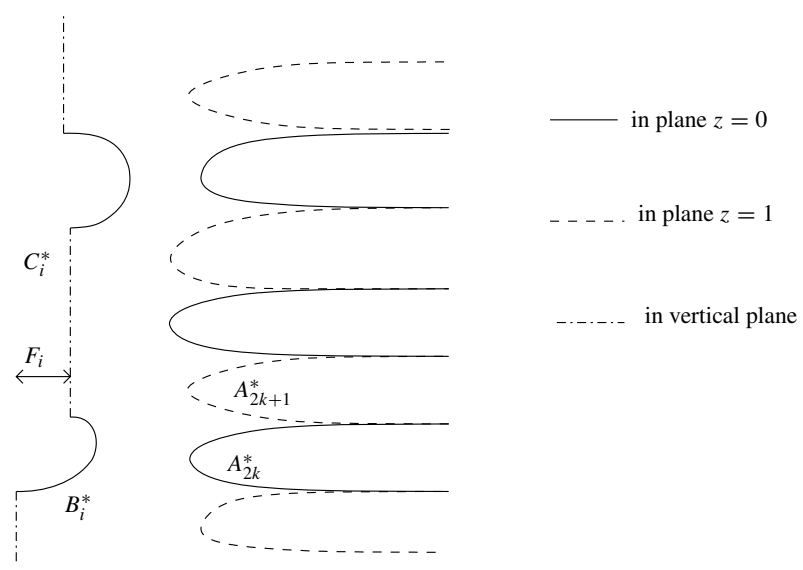

Figure 4. The conjugate minimal surface, Period Problem not solved.

Serrin problem, with the jumps of $u$ at each point $q_{i}$ as parameters. Then we would have to adjust these parameters to guarantee that the conjugate curves $B_{i}^{*}$ all lie in the plane $z=0$. This means another Period Problem to solve. It is automatically solved in our maximal graph approach.

\section{The Period Problem}

In this section, we first formulate the Period Problem in general. Then we solve it provided that $\ell<1$ and there is a finite number of singularities $q_{1}, \ldots, q_{N}$, which are not too close from each other. Our solution to the Period Problem is based on the Poincaré-Miranda Theorem:

Theorem 3 (Poincaré-Miranda). Let $F=\left(F_{1}, \ldots, F_{N}\right)$ be a continuous map from $[0,1]^{N}$ to $\mathbb{R}^{N}$. Write $x=\left(x_{1}, \ldots, x_{N}\right)$. Assume that for each $i, F_{i}(x)$ is negative on the face $x_{i}=0$, while $F_{i}(x)$ is positive on the face $x_{i}=1$. Then there exists $x^{0} \in[0,1]^{N}$ such that $F\left(x^{0}\right)=0$.

4.1. Formulation of the Period Problem. Let $\Omega$ and $\&$ be as in Proposition 1, and let $v$ be the solution of the Dirichlet problem in $\Omega \backslash \&$. Let $u$ be the conjugate function of $v$ and $X^{*}=\left(X_{1}^{*}, X_{2}^{*}, X_{3}^{*}\right)$ be the conjugate minimal surface to the graph of $u$. Both $u$ and $X^{*}$ are only locally well defined, but their differentials are well defined in $\Omega \backslash 8$. Explicitly, $d X^{*}$ is given by

$$
d X_{1}^{*}=\frac{u_{x} u_{y} d x+\left(1+\left(u_{y}\right)^{2}\right) d y}{\sqrt{1+|\nabla u|^{2}}},
$$




$$
\begin{gathered}
d X_{2}^{*}=\frac{-\left(1+\left(u_{x}\right)^{2}\right) d x-u_{x} u_{y} d y}{\sqrt{1+|\nabla u|^{2}}}, \\
d X_{3}^{*}=d v .
\end{gathered}
$$

The Period Problem asks that $X^{*}$ is well defined in $\Omega \backslash \delta$. This is equivalent to $\int_{\gamma} d X^{*}=0$, where $\gamma$ is a small circle around any point of the singular set $\$$.

Proposition 2. $X_{2}^{*}$ and $X_{3}^{*}$ are well defined in $\Omega \backslash \S$.

Proof. This is clear for $X_{3}^{*}$. For $X_{2}^{*}$, we use the following symmetry argument. Let $\tau(x, y)=(x,-y)$. By uniqueness and symmetry of the boundary data, $v \circ \tau=v$. Hence $\tau^{*} d v=d v$, so $v_{x} \circ \tau=v_{x}$ and $v_{y} \circ \tau=-v_{y}$. This gives $u_{x} \circ \tau=-u_{x}$ and $u_{y} \circ \tau=u_{y}$. Hence using (6), $\tau^{*} d X_{2}^{*}=d X_{2}^{*}$. Let $\gamma$ be a small circle around a singularity $q \in$ \&. Since $\tau(\gamma)$ is homologous to $-\gamma$, this gives $\int_{\gamma} d X_{2}^{*}=0$, so $X_{2}^{*}$ is well defined in $\Omega \backslash 8$. This also gives $\tau^{*} d X_{1}^{*}=-d X_{1}^{*}$, so $X_{1}^{*}$ is locally constant on the $x$-axis.

By Proposition 2, we only have to worry about the periods of $d X_{1}^{*}$.

From now on, we assume that $\delta=\left\{q_{1}, \ldots, q_{N}\right\}$ is finite. Let $\gamma_{i}$ be a small circle around the point $q_{i}$ and let

$$
F_{i}\left(q_{1}, \ldots, q_{N}\right)=\int_{\gamma_{i}} d X_{1}^{*}
$$

The Period Problem asks that $F_{i}=0$ for $1 \leq i \leq N$. Note that by symmetry, $F_{i}$ is equal to twice the integral of $d X_{1}^{*}$ on a half circle from $q_{i}+\epsilon$ to $q_{i}-\epsilon$, so $F_{i}=0$ means that the curves $C_{i}^{*}$ and $C_{i-1}^{*}$ are in the same vertical plane as required (see Figure 4).

4.2. Continuity of the periods. To apply the Poincaré-Miranda Theorem, we need the continuity of the periods with respect to the parameters.

Proposition 3. The periods $F_{i}$ depend continuously on $\left(q_{1}, \ldots, q_{N}\right)$.

Proof. Consider an admissible value $\left(q_{1}, \ldots, q_{N}\right)$ of the parameters (namely, all $q_{j}$ satisfy equation (4)). Consider a sequence $\left(q_{1}^{n}, \ldots, q_{N}^{n}\right)$ converging to $\left(q_{1}, \ldots, q_{N}\right)$. Let $\delta_{n}=\left\{q_{1}^{n}, \ldots, q_{N}^{n}\right\}$ and $\delta=\left\{q_{1}, \ldots, q_{N}\right\}$. Let $v_{n}$ and $v$ be the solutions of the Dirichlet problem in $\Omega \backslash \wp_{n}$ and $\Omega \backslash \S$, respectively. Assume the sequence $\left(v_{n}\right)_{n}$ has a divergence line. Then arguing as in the proof of Proposition 1, $L$ has finite length so is a segment connecting two points of $\partial \Omega \backslash \delta$, which contradicts in the same way the fact that the points $q_{j}$ satisfy (4). Hence there are no divergence lines, 
so a subsequence of $\left(v_{n}\right)_{n}$ converges on compact subsets of $\bar{\Omega}$ to a solution to the Dirichlet problem on $\Omega \backslash$ \&. By uniqueness of the solution to this problem, the whole sequence $\left(v_{n}\right)_{n}$ converges to $v$ on compact subsets of $\bar{\Omega}$, and converges smoothly to $v$ on compact subsets of $\Omega \backslash$ s. This implies that $d u_{n}$ converges to $d u$ and $d X_{1, n}^{*}$ converges to $d X_{1}^{*}$ on compact subsets of $\Omega \backslash$ s. Integrating on $\gamma_{i}$ which encloses $q_{i}^{n}$ for big $n$, we obtain that $F_{i}\left(q_{1}^{n}, \ldots, q_{N}^{n}\right) \rightarrow F_{i}\left(q_{1}, \ldots, q_{N}\right)$.

4.3. Local property of the period. From now on we assume that $\ell<1$. Let us define for the rest of the paper

$$
\eta=1-\sqrt{1-\ell^{2}}
$$

In this section, we prove that some properties of the period $F_{i}\left(q_{1}, \ldots, q_{N}\right)$ depends only on the position of $q_{i}$ if the other $q_{j}$ are not too close from $q_{i}$.

Let us denote by $\Omega_{L}$ the box $(-L, L) \times(-\ell, \ell)$ and consider $q \in(-\eta, \eta)$ and a finite set of points $\delta$ in $(-2,-2+\eta) \cup(2-\eta, 2)$. Let $v$ be a solution of the maximal graph equation (2) on $\Omega_{2} \backslash(\{q\} \cup \delta)$, with boundary value $v=\phi$ on $(-2,2) \times\{-\ell, \ell\}$, $v(q)=0$ and $v(\delta)=0$. The boundary value of $v$ on the vertical edges is free, although we require $0 \leq v \leq 1$. Let us study the divergence lines of a sequence of such solutions $v$.

Lemma 4. For every $n \in \mathbb{N}$, let $q_{n}, \wp_{n}$ and $v_{n}$ be as above. We assume that $\lim q_{n}=q$ exists. Then

- if $q \in(-\eta, \eta)$, there is no divergence line in $\Omega_{1} \backslash\{q\}$;

- if $q=\eta$, the only divergence lines meeting $\Omega_{1} \backslash\{\eta\}$ are $\left[\eta, a_{1}^{-}\right]$and $\left[\eta, a_{1}^{+}\right]$.

Proof. The two segments $\left[\eta, a_{1}^{+}\right]$and $\left[\eta, a_{1}^{-}\right]$have length one. Since $v_{n}\left(q_{n}\right)=0$ and $v_{n}\left(a_{1}^{+}\right)=1=v_{n}\left(a_{1}^{-}\right)$, both segments are divergence lines for $\left(v_{n}\right)_{n \in \mathbb{N}}$ in the second case.

Let us now prove that there is no other divergence line in $\Omega_{1}$ for both cases. Since $0 \leq v_{n} \leq 1$ every divergence line is a segment of length at most one. Hence a divergence line $L$ which intersects $\Omega_{1}$ must have an end point in $\bar{\Omega}_{1}$. Because of Lemma 3 these end points needs to be $a_{0}^{ \pm}, a_{-1}^{ \pm}, a_{1}^{ \pm}$or $q$. Let us assume that $a_{0}^{+}$is one end point of $L$. The distance from $a_{0}^{+}$to $\Omega_{2} \backslash \Omega_{1}$ is one, hence the other end point is in $\Omega_{1}$. It can not be $a_{0}^{-}$or $q$ since $v_{n}\left(a_{0}^{+}\right)=v_{n}\left(a_{0}^{-}\right)=\lim v_{n}(q)$. It is not $a_{ \pm 1}^{-}$ since the distance from $a_{0}^{+}$to these points is $\sqrt{1+4 \ell^{2}}>1$. Then $a_{0}^{+}$is not an end point of $L$; by symmetry, this is also true for $a_{0}^{-}$. Let us assume that $a_{-1}^{+}$is an end point of $L$ then the other end point is either $q$ or $a_{1}^{-}$but the distance from $a_{-1}^{+}$to these two points is strictly larger than one since $q \in(-\eta, \eta]$; then $a_{-1}^{+}$is not an end point for $L$. By symmetry, this is also true for $a_{-1}^{-}$and $a_{1}^{ \pm}$unless $q=\eta$ and $L=\left[\eta, a_{1}^{ \pm}\right]$ 
which is the case we studied first. Then we can assume that $q$ is an end point of $L$ and the other one is in $\Omega_{2} \backslash \Omega_{1}$. By Lemma 3, if $L$ is not horizontal, the second end point is on the vertical edges of $\Omega_{2}$ but the distance from $q$ to these edges is larger than $2-\eta>1$. So $L$ is horizontal; we assume, for example, that $L$ is on the left of $q$. Since the length of $L$ is less than 1 , the other end point of $L$ needs to be in the interior of $\Omega_{2}$ and $s_{n} \cap(2-\eta, 2] \neq \emptyset$. Let $s_{n}=\min s_{n} \cap(2-\eta, 2]$. We assume that $\left(s_{n}\right)$ converges to $s$ in $[2-\eta, 2]$, then $L$ is the segment $[q, s]$. We have $v_{n}\left(s_{n}\right)=0$. Since $L$ is a divergence line $|L|=\lim \left|v_{n}(q)-v_{n}(s)\right|=\lim \left|v_{n}\left(q_{n}\right)-v_{n}\left(s_{n}\right)\right|=0$ this gives a contradiction and the lemma is proved.

Let $v$ be a solution of the maximal graph equation (2) on $\Omega_{2} \backslash(\{q\} \cup 8)$ as above, besides we assume now the symmetry $v(x, y)=v(x,-y)$. In applications, $v$ will be the restriction of some $v\left[q_{1}, \ldots, q_{n}\right]$ to a box around one $q_{i}$. Let $\gamma$ be a small circle around $q$ then we define the period $F(v)$ by $\int_{\gamma} d X_{1}^{*}$ where $d X_{1}^{*}$ is given by equation (5) with $u$ the conjugate function to $v$. We then have some control on the behaviour of the period.

Proposition 4. There exists $\eta_{0} \in(0, \eta)$ which depends only on $\ell$ such that for any solution $v$ of the above Dirichlet problem on $\Omega_{2} \backslash(\{q\} \cup \delta)$ we have:

- if $\eta_{0} \leq q<\eta, F(v) \geq 1$;

- if $-\eta<q \leq-\eta_{0}, F(v) \leq-1$.

Proof. Let $\sigma(x, y)=-(x, y)$ and $v$ be a solution of the above Dirichlet problem on $\Omega_{2} \backslash(\{q\} \cup 8)$ then $v^{\prime}=v \circ \sigma$ is a solution of this Dirichlet problem on $\Omega_{2} \backslash(\{-q\} \cup-\wp)$. From the definition of $d u=d \Phi_{v}$ and $d u^{\prime}=d \Phi_{v^{\prime}}$, we obtain $\sigma^{*} d u=d u^{\prime}$. From equation (5), we get $\sigma^{*} d X_{1}^{*}=-d X_{1}^{* \prime}$ where $d X_{1}^{*}$ and $d X_{1}^{* \prime}$ are respectively associated to $v$ and $v^{\prime}$. Since $\sigma$ preserves orientation, integrating on a small circle around $q$ gives $F(v)=-F\left(v^{\prime}\right)$. Thus the second item of the proposition is a consequence of the first one.

If the first item is wrong there exists a sequence $q_{n} \rightarrow \eta$ and for each $n$ a set $s_{n}$ and a solution $v_{n}$ of the above Dirichlet problem on $\Omega_{2} \backslash\left(\left\{q_{n}\right\} \cup \wp_{n}\right)$ such that $F\left(v_{n}\right)<1$. Let us prove that, actually, $\lim F\left(v_{n}\right)=\infty$.

By Lemma 4 the two segments $L^{+}=\left[\eta, a_{1}^{+}\right]$and $L^{-}=\left[\eta, a_{1}^{-}\right]$are divergence lines for $\left(v_{n}\right)_{n \in \mathbb{N}}$. On $L^{+},\left(\nabla v_{n}\right)_{n \in \mathbb{N}}$ converges to $\overrightarrow{\eta a_{1}^{+}}=\left(\sqrt{1-\ell^{2}}, \ell\right)$. Let $\Omega_{-}$ be the connected component of $\Omega_{1} \backslash\left(L^{+} \cup L^{-}\right)$containing the origin. Because of Lemma $4, \Omega_{-}$is included in the convergence domain of $\left(v_{n}\right)_{n \in \mathbb{N}}$

Then we can assume that the sequence $\left(v_{n}\right)_{n \in \mathbb{N}}$ converges on $\Omega_{-}$to a solution $v$ which takes on the boundary the value $\phi$ on $\partial \Omega \cap \Omega_{-}$and $|p-\eta|$ for $p \in L^{+} \cup L^{-}$. For every $n$, let $u_{n}$ be the conjugate function $\Phi_{v_{n}}$ which is defined on $\Omega_{-} \backslash\left[q_{n}, \eta\right]$. The limit domain of $\left(\Omega_{-} \backslash\left[q_{n}, \eta\right]\right)_{n \in \mathbb{N}}$ is $\Omega_{-}$and $\left(u_{n}\right)_{n \in \mathbb{N}}$ converges to $u=\Phi_{v}$. Because of the boundary value of $v, u$ takes the value $+\infty$ along $L^{+}$. We are interested in 
what happens near the middle point $((1+\eta) / 2, \ell / 2)$ of $L^{+}$. Because of Lemma 1 in [3] we have

$$
\frac{u_{y}}{\sqrt{1+|\nabla u|^{2}}}\left(\frac{1+\eta}{2}, y\right) \longrightarrow-\sqrt{1-\ell^{2}}
$$

when $y \rightarrow \ell / 2$ with $y>\ell / 2$. Lemma 1 in [3] implies also that

$$
u_{y}\left(\frac{1+\eta}{2}, y\right) \leq-\frac{C}{|y-\ell / 2|}, \quad C>0
$$

for $y>\ell / 2$ near $\ell / 2$.

Consider the following path $\Gamma$ : it is the union of the segment $[((1+\eta) / 2,0)$, $((1+\eta) / 2,3 \ell / 4)]$ with a curve $\Gamma_{3}$ in $\Omega_{-} \cap\{y>0\}$ that joins $((1+\eta) / 2,3 \ell / 4)$ to $(0,0)$. For $t \in(\ell / 2,3 \ell / 4)$, let $\Gamma_{1}(t)$ be the segment $[((1+\eta) / 2,0),((1+\eta) / 2, t)]$ and $\Gamma_{2}(t)$ be the segment $[((1+\eta) / 2, t),((1+\eta) / 2,3 \ell / 4)]$.
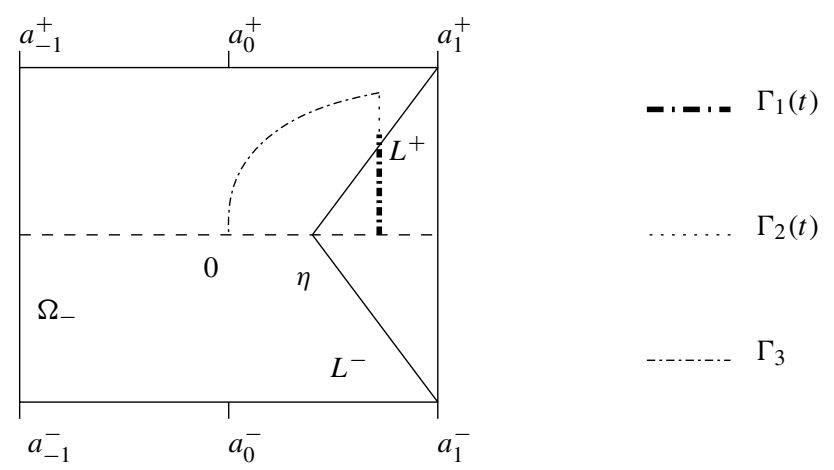

Figure 5

Because of the symmetry $v_{n}(x, y)=v_{n}(x-y)$, for large $n$, the period $F\left(v_{n}\right)$ is given by $2 \int_{\Gamma} d X_{1, n}^{*}$ where $d X_{1, n}^{*}$ is associated to $u_{n}$. Because of Equation (5), $\int_{\Gamma_{1}(t)} d X_{1, n}^{*} \geq 0$. Hence

$$
F\left(v_{n}\right) \geq 2 \int_{\Gamma_{2}(t) \cup \Gamma_{3}} d X_{1, n}^{*}
$$

Since the convergence $u_{n} \rightarrow u$ is smooth on compact subsets of $\Omega_{-}$,

$$
\int_{\Gamma_{2}(t) \cup \Gamma_{3}} d X_{1, n}^{*} \longrightarrow \int_{\Gamma_{2}(t) \cup \Gamma_{3}} d X_{1}^{*}
$$

with $d X_{1}^{*}$ associated to $u$. By (7) and (8), we have

$$
\int_{\Gamma_{2}(t)} d X_{1}^{*} \underset{t \rightarrow \frac{\ell}{2}^{+}}{\longrightarrow}+\infty \text {. }
$$


Equations (9), (10) and (11) imply that $\lim F\left(v_{n}\right)=+\infty$. The lemma is proved.

4.4. Solution of the Period Problem. In this section, we go back to the $N$-dimensional Period Problem. Consider $N$ integers $p_{1}<\cdots<p_{N}$ (these will specify "where" we want to put the handles). Recall that $\ell<1$ and $\eta=1-\sqrt{1-\ell^{2}}$ and that Lemma 4 gives us a number $\eta_{0}<\eta$. Equation (4) means that each singular point $q_{i}$ must be at distance less than $\eta$ from an even integer. We require that $\left|q_{i}-2 p_{i}\right|<\eta$ for $i=1, \ldots, N$. The next proposition solves the Period Problem with this setting.

Proposition 5. For any $N$ and for any choice of $p_{1}, \ldots, p_{N}$ satisfying $p_{1}<p_{2}<$ $\cdots<p_{N}$, there exists $\left(q_{1}, \ldots, q_{N}\right)$ satisfying $\left|q_{i}-2 p_{i}\right|<\eta_{0}$ and $F_{i}\left(q_{1}, \ldots, q_{N}\right)=$ 0 for $i=1, \ldots, N$.

Proof. Consider any $p_{1}, \ldots, p_{N}$ such that $p_{1}<\cdots<p_{N}$. Consider any value of the parameters $\left(q_{1}, \ldots, q_{N}\right)$ in the cubic box defined by $q_{i} \in\left[2 p_{i}-\eta_{0}, 2 p_{i}+\eta_{0}\right]$. Consider some $i, 1 \leq i \leq N$. Translating by $-2 p_{i}$, Proposition 4 tells us that if $q_{i}=2 p_{i}+\eta_{0}, F_{i}\left(q_{1}, \ldots, q_{N}\right) \geq 1$ while if $q_{i}=2 p_{i}-\eta_{0}, F_{i}\left(q_{1}, \ldots, q_{N}\right) \leq$ -1 . The result then follows from the Poincaré-Miranda Theorem since $F_{i}$ depends continuously in the $q_{j}$.

Remark 3. We do not know if the solution to the Period Problem is unique. Since we do not know how to compute derivatives of the periods with respect to the parameters, its seems hard to obtain uniqueness.

\section{Finite genus}

Proposition 5 implies the following

Corollary 1. For each $N \geq 1$, there exists a complete, properly embedded minimal surface in $\mathbb{R}^{2} \times \mathbb{S}^{1}$ which has genus $N$, infinite total curvature, infinitely many ends, and two limit ends.

Proof. Consider integers $p_{1}<\cdots<p_{N}$. Let $\left(q_{1}, \ldots, q_{N}\right)$ be the solution to the Period Problem given by Proposition 5. Let $v=v\left[q_{1}, \ldots, q_{N}\right], u=u\left[q_{1}, \ldots, q_{N}\right]$ and $X^{*}=X^{*}\left[q_{1}, \ldots, q_{N}\right]$. Then $X^{*}$ is well defined in $\Omega \backslash\left\{q_{1}, \ldots, q_{N}\right\}$. To see that the image of $X^{*}$ is embedded we argue as follows. Let $M$ be the graph of $u$ on the strip $\Omega^{+}=\mathbb{R} \times(0, \ell)$ and $M^{*}$ be the conjugate minimal surface to $M$, so $M^{*}=X^{*}\left(\Omega^{+}\right)$. Since $\Omega^{+}$is convex, $M^{*}$ is a graph over a planar domain by the Theorem of R. Krust, so is embedded.

Since the Period Problem is solved, all segments $\left(q_{i}, q_{i+1}\right), i=1, \ldots, N-1$, as well as the half lines $\left(-\infty, q_{1}\right)$ and $\left(q_{N},+\infty\right)$, are mapped onto geodesics in the vertical plane $x=0$ (after a suitable translation). Consider now some $(x, y) \in \Omega^{+}$ 
such that $x \neq q_{i}$ for all $i$. From the formula (5) we see that $\int_{(x, 0)}^{(x, y)} d X_{1}^{*}>0$. Hence the point $X^{*}(x, y)$ lies in $x>0$. By continuity this is true for all points of $\Omega^{+}$, so $M^{*}$ is an embedded minimal surface in $x>0,0<z<1$. Extending by symmetry with respect to the plane $x=0$ and the horizontal planes at integer heights we obtain an embedded, periodic minimal surface in $\mathbb{R}^{3}$ with period $T=(0,0,2)$. The quotient by this period is an embedded minimal surface $\mathcal{M}_{N}$ in $\mathbb{R}^{2} \times \mathbb{S}^{1}$ of genus $N$ (now and in the following, we identify $\mathbb{S}^{1}$ with $\mathbb{R} / 2 \mathbb{Z}$ ). We will see in Section 7 that it has bounded curvature. By a theorem of Meeks - Rosenberg, theorem 2.1 in [14], a complete embedded minimal surface in $\mathbb{R}^{3}$ with bounded curvature is properly embedded. Hence $\mathcal{M}_{N}$ is properly embedded.

In the following, when $\left(q_{1}, \ldots, q_{N}\right)$ is given by Proposition 5 , the associated minimal surface given by the above corollary will be denoted by $\mathcal{M}\left[q_{1}, \ldots, q_{N}\right]$. This surface is normalized so that the conjugate to the point $(-1,0, u(-1,0))$ is the point $(0,0, v(-1,0))$.

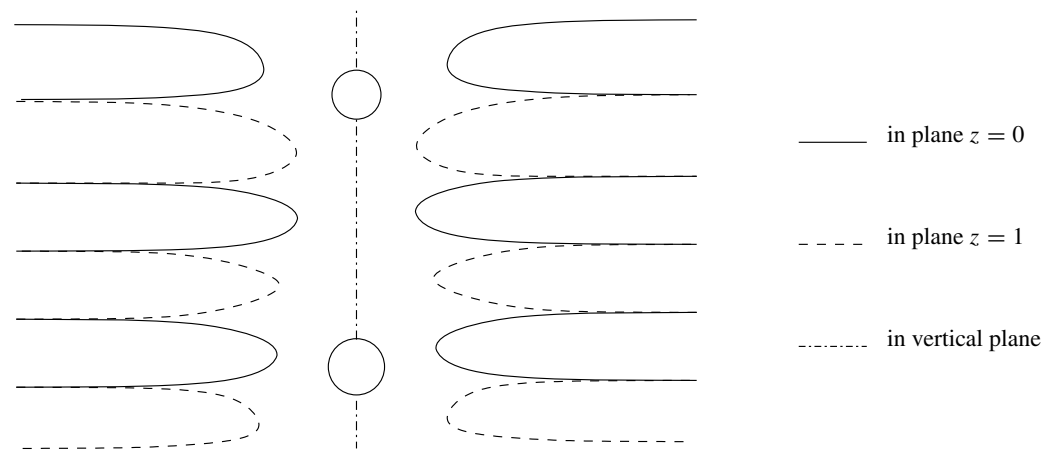

Figure 6. The conjugate surface, Period Problem solved.

Remark 4. In Proposition 5, if the singularity set $\delta$ is empty, the period Problem is solved. Then a surface $\mathcal{M}[\emptyset]$ of genus zero exists; in fact this surface is a Karcher's toroidal halfplane layer.

\section{Infinite genus}

In this section, we consider the case where we have an infinite number of singularities.

Proposition 6. Let $\ell$ and $\eta_{0}$ be as in Proposition 5. Consider a strictly increas-

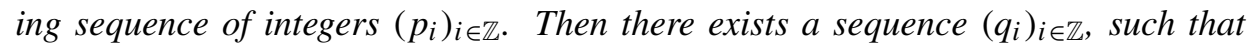
$\left|q_{i}-2 p_{i}\right| \leq \eta_{0}$, which solves the Period Problem $F_{j}\left(q_{i}: i \in \mathbb{Z}\right)=0$ for all $j \in \mathbb{Z}$. 
Proof. Consider some $n \in \mathbb{N}$. We apply Proposition 5 with $N=2 n+1$ and the $N$ integers $p_{-n}, \ldots, p_{n}$, and we obtain $N$ real numbers $q_{-n, n}, \ldots, q_{n, n}$ such that $\left|q_{i, n}-2 p_{i}\right| \leq \eta_{0}$ for all $|i| \leq n$, and $F_{j}\left(q_{i, n}:|i| \leq n\right)=0$ for all $|j| \leq n$. Using a diagonal process, we can find a subsequence (which we will still denote by $n$ ) such that $q_{i}=\lim _{n \rightarrow \infty} q_{i, n}$ exists for all for all $i \in \mathbb{Z}$. The limit domain of $\left(\Omega \backslash\left\{q_{i, n}:|i| \leq n\right\}\right)_{n \in \mathbb{N}}$ is $\Omega \backslash\left\{q_{i}: i \in \mathbb{Z}\right\}$. Let $v_{n}=v\left[q_{i, n}:|i| \leq n\right]$ and $v_{\infty}=$ $v\left[q_{i}: i \in \mathbb{Z}\right]$. Arguing as usual, the sequence $\left(v_{n}\right)_{n \in \mathbb{N}}$ has no divergence line, so up to a subsequence, it converges on compact subsets of $\bar{\Omega}$ to a solution $v$ of the Dirichlet problem on $\Omega \backslash\left\{q_{i}: i \in \mathbb{Z}\right\}$. By uniqueness, $v=v_{\infty}$. Then $d X_{1}^{*}\left[q_{i, n}:|i| \leq n\right]$ converges to $d X_{1}^{*}\left[q_{i}: i \in \mathbb{Z}\right]$ on compact subsets of $\Omega \backslash\left\{q_{i}: i \in \mathbb{Z}\right\}$. Integrating on $\gamma_{j}$ gives

$$
F_{j}\left(q_{i}: i \in \mathbb{Z}\right)=\lim _{n \rightarrow \infty} F_{j}\left(q_{i, n}:|i| \leq n\right)=0 .
$$

In the following when a sequence $\left(p_{i}\right)_{i \in \mathbb{Z}}$ satisfies the hypotheses of the above proposition and $\left(q_{i}\right)_{i \in \mathbb{Z}}$ is a sequence such that $\left|q_{i}-2 p_{i}\right| \leq \eta_{0}$ for all $i \in \mathbb{Z}$ and $F_{j}\left(q_{i}: i \in \mathbb{Z}\right)=0$ for all $j \in \mathbb{Z}$, we shall say that $\left(q_{i}\right)_{i \in \mathbb{Z}}$ solves the Period Problem for the data $\left(p_{i}\right)_{i \in \mathbb{Z}}$.

Corollary 2. For any strictly increasing sequence of integers $\left(p_{i}\right)_{i \in \mathbb{Z}}$, there exists a properly embedded minimal surface $\mathcal{M}$ in $\mathbb{R}^{2} \times \mathbb{S}^{1}$ which has infinite genus, infinite total curvature, infinitely many ends, and two limit ends. Moreover, if the sequence $\left(p_{i+1}-p_{i}\right)_{i \in \mathbb{Z}}$ is not periodic, then $\mathcal{M}$ is not periodic.

Proof. Same as proof of Corollary 1.

As above when $\left(q_{i}\right)_{i \in \mathbb{Z}}$ solves the Period Problem for the data $\left(p_{i}\right)_{i \in \mathbb{Z}}$, the associated surface is denoted by $\mathcal{M}\left[q_{i}: i \in \mathbb{Z}\right]$ and is normalized as in the finite genus case.

Using the notation of the proof of Proposition 6, we define $\mathcal{M}_{N}=\mathcal{M}\left[q_{i, N}\right.$ : $-N \leq i \leq N]$ and $\mathcal{M}=\mathcal{M}\left[q_{i}: i \in \mathbb{Z}\right]$.

Proposition 7. A subsequence of $\left(\mathcal{M}_{N}\right)_{N}$ converges smoothly on compact subsets of $\mathbb{R}^{2} \times \mathbb{S}^{1}$ to $\mathcal{M}$.

Proof. Since a subsequence of $\left(v_{n}\right)_{n}$ converges to $v$, the result seems to be obvious. This is not immediate for the following reason: the convergence of the conjugate functions $\left(u_{n}\right)_{n}$ to $u$ only holds on compact subsets of $\Omega \backslash\left\{q_{i}: i \in \mathbb{Z}\right\}$. In particular, this convergence does not tell us anything for the graph of $u$ above the singular points and the vertices $a_{k}^{+}, k \in \mathbb{Z}$. Since these correspond to the horizontal symmetry curves on the conjugate minimal surface, we see that the convergence of $\left(v_{n}\right)_{n}$ to $v$ is not enough to conclude. 
One way around this difficulty is as follows: we will see in the next section that the curvature of $\mathcal{M}_{N}$ is bounded by a constant independent of $N$. By the Regular Neighborhood Theorem, or "Rolling Lemma" (firstly proven by A. Ros [15], Lemma 4, for properly embedded minimal surfaces in $\mathbb{R}^{3}$ with finite total curvature, and generalized to properly embedded minimal surfaces with bounded curvature by Meeks and Rosenberg [14], Theorem 5.3), each $\mathcal{M}_{N}$ has an embedded tubular neighborhood of radius $1 / \sqrt{c}$. In particular, we have local area bounds, namely the area of $\mathcal{M}_{N}$ inside a ball of radius $1 / \sqrt{c}$ is bounded by some constant. By standard results, a subsequence of $\left(\mathcal{M}_{N}\right)_{N}$ converges smoothly on compact subsets of $\mathbb{R}^{2} \times \mathbb{S}^{1}$ to a limit minimal surface $\mathcal{M}_{\infty}$. Since $\left(v_{n}\right)_{n}$ converges to $v$, the limit $\mathcal{M}_{\infty}$ needs to be $\mathcal{M}$.

Here is another result in the same spirit, which will be useful in Section 8. For every $n \in \mathbb{N}$, let $\left(p_{i, n}\right)_{i \in \mathbb{Z}}$ be a sequence as in Proposition 6, namely $\left(p_{i, n}\right)_{i \in \mathbb{Z}}$ is a strictly increasing sequence of integers. Let $\left(q_{i, n}\right)_{i \in \mathbb{Z}}$ be a sequence that solves the Period Problem for the data $\left(p_{i, n}\right)_{i \in \mathbb{Z}}$.

Proposition 8. Let $\left(p_{i, n}\right)_{i \in \mathbb{Z}}$ and $\left(q_{i, n}\right)_{i \in \mathbb{Z}}$ be defined as above. Let us assume that for every $i \in \mathbb{Z}, \lim _{n} p_{i, n}=p_{i, \infty}$ and $\lim _{n} q_{i, n}=q_{i, \infty}$. Then $\left(q_{i, \infty}\right)_{i \in \mathbb{Z}}$ solves the Period Problem for the data $\left(p_{i, \infty}\right)_{i \in \mathbb{Z}}$ and $\left(\mathcal{M}\left[q_{i, n}: i \in \mathbb{Z}\right]\right)_{n \in \mathbb{N}}$ converges smoothly on compact subsets of $\mathbb{R}^{2} \times \mathbb{S}^{1}$ to $\mathcal{M}\left[q_{i, \infty}: i \in \mathbb{Z}\right]$.

Proof. Let $v_{n}$ be $v\left[q_{i, n}: i \in \mathbb{Z}\right]$. First we notice that the convergence of $\left(q_{i, n}\right)_{n \in \mathbb{Z}}$ implies the convergence of $\left(p_{i, n}\right)_{n} \in \mathbb{Z}$. Since we have $p_{i+1, n}-p_{i, n} \geq 1$ and $\left|q_{i, n}-2 p_{i, n}\right| \leq \eta_{0}$ for all $i$ and $n$, we get $p_{i+1, \infty}-p_{i, \infty} \geq 1$ and $\left|q_{i, \infty}-2 p_{i, \infty}\right| \leq \eta_{0}$.

Since all the $\left(q_{i, n}\right)_{n \in \mathbb{N}}$ converge, the limit domain of $\left(\Omega \backslash\left\{q_{i, n}: i \in \mathbb{Z}\right\}\right)_{n \in \mathbb{N}}$ is $\Omega \backslash\left\{q_{i, \infty}: i \in \mathbb{Z}\right\}$. As in the preceding, the sequences $\left(v_{n}\right)_{n \in \mathbb{N}}$ has no divergence line and converges to a solution $v_{\infty}$ of the Dirichlet problem on the limit domain $\Omega \backslash\left\{q_{i, \infty}: i \in \mathbb{Z}\right\}$. By uniqueness, $v_{\infty}=v\left[q_{i, \infty}: i \in \mathbb{Z}\right]$. As in the proof of Proposition 6,

$$
F_{j}\left(q_{i, \infty}: i \in \mathbb{Z}\right)=\lim _{n \rightarrow \infty} F_{j}\left(q_{i, n}: i \in \mathbb{Z}\right)=0 .
$$

Then $\left(q_{i, \infty}\right)_{i \in \mathbb{Z}}$ solves the Period Problem.

Now as in the proof of Proposition 7, since the curvature of the surfaces $\mathcal{M}\left[q_{i, n}\right.$ : $i \in \mathbb{Z}]$ is uniformly bounded (see Proposition 10), the sequence $\left(\mathcal{M}\left[q_{i, n}: i \in \mathbb{Z}\right]\right)_{n \in \mathbb{N}}$ converges smoothly on compact subsets of $\mathbb{R}^{2} \times \mathbb{S}^{1}$ to a limit minimal surface $\mathcal{M}_{\infty}$. Since $\left(v_{n}\right)_{n \in \mathbb{N}}$ converges to $v\left[q_{i, \infty}: i \in \mathbb{Z}\right]$, the surfaces $\mathcal{M}_{\infty}$ needs to be $\mathcal{M}\left[q_{i, \infty}\right.$ : $i \in \mathbb{Z}]$. 


\section{Bounded curvature}

In this section we prove that the curvature of the surfaces $\mathcal{M}$ given by Corollaries 1 and 2 are bounded by a constant $C$ depending only on $\ell$. Actually, because of Proposition 7, it suffices to prove it in the finite genus case.

7.1. Size of the handles. Suppose that $v$ is the solution of the Dirichlet problem on $\Omega \backslash\left\{q_{1}, \ldots, q_{N}\right\}$. The multi-valuation of its conjugate function $u$ around the singularity $q_{i}$ is $\int_{\gamma_{i}} d u$. This is equal to twice the length of the vertical segment $B_{i}$, which is equal to the length of the geodesic $B_{i}^{*}$. So the multi-valuation of $u$ may be understood as the "size" of the handle. In this section we give a uniform lower bound for this multi-valuation, which prevents the handles from getting too small.

We use the notation $\Omega_{L}=(-L, L) \times(-\ell, \ell)$.

Proposition 9. Consider some $\ell<1$ and some $\eta_{0}<\eta$. There exists $\kappa>0$ (depending on $\ell$ and $\left.\eta_{0}\right)$ such that the following is true: Let $q \in\left(-\eta_{0}, \eta_{0}\right)$ and $s \subset(-2,-2+\eta) \cup(2-\eta, 2)$. Let $v$ be a solution of the maximal graph equation (2) in $\Omega_{2} \backslash(\{q\} \cup 8)$ with boundary value $\varphi$ on $[-2,2] \times\{-\ell, \ell\}$ and 0 at $\{q\} \cup 8$. As in Lemma 4 the boundary value on the vertical edges is free, but we require $v$ to be between 0 and 1 . Let $u$ be the conjugate function of $v$. Let $\gamma$ be a small circle around $q$. Then $\left|\int_{\gamma} d u\right| \geq \kappa$.

Proof. Assume by contradiction that the proposition is not true. Then there exists sequences $\left(q_{n}\right)_{n}$ and $\left(\delta_{n}\right)_{n}$ and a sequence $\left(v_{n}\right)_{n}$ such that $\int_{\gamma} d u_{n} \rightarrow 0$. Passing to a subsequence, $q_{n}$ converges to some $q \in\left[-\eta_{0}, \eta_{0}\right] \subset(-\eta, \eta)$. By Lemma 4 , the sequence $\left(v_{n}\right)_{n}$, restricted to $\Omega_{1} \backslash\{q\}$, does not have any divergence line, so passing to a subsequence, it converges to a solution $v$ on $\Omega_{1}$. Then the conjugate differential $d u_{n}$ of $v_{n}$ converges on compact subsets of $\Omega_{1} \backslash\{q\}$ to the conjugate differential $d u$ of $v$. This implies that $\int_{\gamma} d u=0$, so $u$ is in fact well defined in $\Omega_{1} \backslash\{q\}$. Since it satisfies the minimal graph equation, the point $q$ is a removable singularity, so $u$ extends smoothly to $q$. But then $v$ itself also extends smoothly to $q$. Since $v(q)=0$ and $0 \leq v \leq 1$, the maximum principle for maximal surfaces gives us that $v=0$ in $\Omega_{1}$; this contradicts $v=\varphi$ on the boundary.

7.2. Gradient estimates. Recall that the graph of $u$ is bounded by a vertical segment above each singularity $q_{i}$. Along this segment, the normal is horizontal. The following lemma ensures that the normal remains close to the horizontal on the disk $D\left(q_{i}, \delta\right)$, where $\delta$ is a number we can control in function of the length of the vertical segment.

Lemma 5. For any $C>0$, for any $\kappa>0$, there exists $\delta>0$ such that the following is true: let $v$ be any solution of the maximal graph equation (2) on the punctured disk $D(0,1) \backslash\{0\}$ with a singularity at the origin. Assume that $v(0)=0$ and $0 \leq v \leq 1$. 
Let $d u$ be the conjugate differential of $v$. Assume that $\left|\int_{\gamma} d u\right| \geq \kappa$. Then $|\nabla u| \geq C$ in $D(0, \delta) \backslash\{0\}$.

Proof. Assume by contradiction that the lemma is false. Then there exists $C>0$, $\kappa>0$, and sequences $\left(v_{n}\right)_{n},\left(p_{n}\right)_{n}$, such that $v_{n}$ is a solution of (2) in $D(0,1) \backslash\{0\}$, $p_{n} \rightarrow 0, \int_{\gamma} d u_{n} \geq \kappa$ and $\left|\nabla u_{n}\left(p_{n}\right)\right| \leq C$. Let $\lambda_{n}=\left|p_{n}\right|$. Let $\tilde{v}_{n}(p)=v_{n}\left(\lambda_{n} p\right) / \lambda_{n}$ (so the graph of $\tilde{v}_{n}$ is the graph of $v_{n}$ scaled by $1 / \lambda_{n}$ ). Let $\tilde{p}_{n}=p_{n} / \lambda_{n}$; by rotation we may assume that $\tilde{p}_{n}=(1,0)$. Then $\tilde{v}_{n}$ solves (2) in the punctured disk of radius $1 / \lambda_{n}$. This domain converges to the plane punctured at the origin.

Let us study the convergence of the sequence $\left(\tilde{v}_{n}\right)_{n}$. If there are no divergence lines, then the sequence $\left(\tilde{v}_{n}\right)_{n}$ converges on compact subsets of the punctured plane to a solution $\tilde{v}$. Then the conjugate differentials $d \tilde{u}_{n}$ converge to $d \tilde{u}=d \Phi_{\tilde{v}}$. However,

$$
\left|\int_{\gamma} d \tilde{u}_{n}\right|=\frac{1}{\lambda_{n}}\left|\int_{\gamma} d u_{n}\right| \geq \frac{\kappa}{\lambda_{n}} \rightarrow \infty
$$

so $\int_{\gamma} d \tilde{u}=\infty$, which is absurd. So there must be divergence lines.

Observe that

$$
\left|\nabla \tilde{v}_{n}(1,0)\right|=\left|\nabla \tilde{v}_{n}\left(\tilde{p}_{n}\right)\right|=\left|\nabla v_{n}\left(p_{n}\right)\right|=\frac{\left|\nabla u_{n}\left(p_{n}\right)\right|}{\sqrt{1+\left|\nabla u_{n}\left(p_{n}\right)\right|^{2}}} \leq \frac{C}{\sqrt{1+C^{2}}}<1 .
$$

Hence the point $(1,0)$ is in the convergence domain of the sequence $\tilde{v}_{n}$. Let $U$ be the component of the convergence domain containing the point $(1,0)$. Since $\tilde{v}_{n} \geq 0$, a divergence line cannot extend infinitely in both directions, so must be a half-line ending at the origin. If there are at least two divergence lines then $U$ is a sector defined in polar coordinates by $0<r<\infty, \alpha_{1}<\theta<\alpha_{2}$. The conjugate functions $\tilde{u}_{n}$ are well defined in $U$ and converge to $\tilde{u}$. Then $\tilde{u}$ takes the values $\pm \infty$ on the half-lines $\theta=\alpha_{1}$ and $\theta=\alpha_{2}$. Since $\tilde{v}(0)=0$ and $\tilde{v} \geq 0, \tilde{u}$ takes the values $+\infty$ on $\theta=\alpha_{1}$ and $-\infty$ on $\theta=\alpha_{2}$. It is proven in [8], Proposition 2, that this Jenkins-Serrin problem has no solution.

If there is only one divergence line, then $U$ is a sector of angle $2 \pi$ defined in polar coordinates by $0<r<\infty, \alpha<\theta<\alpha+2 \pi$. Then $\tilde{u}$ solves the following Jenkins-Serrin problem: $u=+\infty$ on the half-line $\theta=\alpha$ (approaching this line with $\theta>\alpha$ ) and $u=-\infty$ on $\theta=\alpha+2 \pi$ (approaching with $\theta<\alpha+2 \pi$. It is proven in [8], Proposition 4, that this Jenkins- Serrin problem has no solution. This contradiction proves the lemma.

The following lemma provides a similar estimate in a neighborhood of the boundary points $a_{k}^{+}, k \in \mathbb{Z}$. It is proven in [6], Lemma 6 .

Lemma 6. Given $C>0$, there exists $\delta>0$ such that the following is true: let $u$ be a solution of the minimal graph equation (1) in the half disk $D(0,1) \cap\{y>0\}$, 
with boundary values $+\infty$ on the segment $(0,1) \times\{0\}$ and $-\infty$ on the segment $(-1,0) \times\{0\}$. Let $v$ be the conjugate function of $u$. Assume that $v(0)=0$ and $v \geq 0$. Then $|\nabla u| \geq C$ in $D(0, \delta) \cap\{y>0\}$.

7.3. Curvature estimate. Let $\mathcal{M}$ be a minimal surface given by Corollary 1 .

Proposition 10. There exists a constant $C$ (depending only on $\ell$ ) such that the Gauss curvature $K$ of the surface $\mathcal{M}$ is bounded by $C$.

Proof. Since $M^{*}$ and $M$ are locally isometric, it suffices to bound the curvature of $M$. The proof is based on stability arguments. In what follows, all constants involved only depend on $\ell$.

Recall that $M$ is the graph of $u$ on $\Omega^{+}=\mathbb{R} \times(0, \ell)$. Let $q_{1}, \ldots, q_{N}$ be given by Proposition 5. By Proposition 9, there exists $\kappa$ such that $\left|\int_{\gamma_{i}} d u\right| \geq \kappa$ for $i=1, \ldots, N$. We apply Lemma 5 with $C=100$ and obtain a $\delta_{1}<\ell$ such that $|\nabla u| \geq 100$ in $D\left(q_{i}, \delta_{1}\right), i=1, \ldots, N$. We apply Lemma 6 with again $C=100$ and obtain a $\delta_{2}<\ell$ such that $|\nabla u| \geq 100$ in $D\left(a_{k}^{+}, \delta_{2}\right), k \in \mathbb{Z}$. We take $\delta=\min \left\{\delta_{1}, \delta_{2}\right\}$. Fix some $i=1, \ldots, N$. Let $U$ be the graph of $u$ above the half disk $D\left(q_{i}, \delta\right) \cap \Omega^{+}$. Since $|\nabla u| \geq 100$, the Gauss image of $U$ is included in the spherical domain $\mathbb{S}^{2} \cap\{|z| \leq 1 / 100\}$. The boundary of $U$ consists of a vertical segment, two horizontal segments and a helix-like looking curve which is a graph on $\mathbb{S}^{1}\left(q_{i}, \delta\right) \cap \Omega^{+}$. Completing by all symmetries, we obtain a minimal surface $\Sigma$ which is bounded by two helix-like looking curves, and which is complete in the cylinder $D\left(q_{i}, \delta\right) \times \mathbb{R}$. The surface $\Sigma$ is of course not a graph anymore. However its Gauss image is still included in $\mathbb{S}^{2} \cap\{|z|<1 / 100\}$. As the spherical area of this domain is less than $2 \pi, \Sigma$ is stable by the theorem of Barbosa Do Carmo [1]. Consider now a point $(x, y) \in D\left(q_{i}, \delta / 2\right)$ and let $p=(x, y, u(x, y))$ be the corresponding point on $M$. Since $p \in \Sigma$ is at distance more than $\delta / 2$ from the boundary of $\Sigma$, the theorem of Schoen [17] ensures that the Gauss curvature at $p$ is bounded by $c /(\delta / 2)^{2}$ for some universal constant $c$. The same argument gives the same estimate for the Gauss curvature when $(x, y) \in D\left(a_{k}^{+}, \delta / 2\right), k \in \mathbb{Z}$.

Assume now that $(x, y) \in \Omega^{+}$is at distance more than $\delta / 2$ from all points $q_{i}$ and all points $a_{k}^{+}$. Let again $p=(x, y, u(x, y))$. If $y>\delta / 4$, then the distance of $p$ to the boundary of $M$ is greater than $\delta / 4$ (because $u= \pm \infty$ on the top edges). Since $M$ is a graph, it is stable, so the Gauss curvature at $p$ is bounded by $c /(\delta / 4)^{2}$.

It remains to understand the case $0<y<\delta / 4$. There exists $i$ such that $q_{i}<$ $x<q_{i+1}$ (with the convention that $q_{0}=-\infty$ and $q_{N+1}=+\infty$ ). Consider the box $\left(q_{i}, q_{i+1}\right) \times(-\delta / 2, \delta / 2)$. As this is a simply connected domain of $\Omega, u$ is well defined on it. Let $V$ be the graph of $u$ on this box. The distance of $p=(x, y, u(x, y))$ to the boundary of $V$ is greater than $\delta / 4$. Since $V$ is stable, we conclude again that the Gauss curvature at $p$ is bounded by $c /(\delta / 4)^{2}$. 


\section{Quasi-periodicity}

In this section, we prove that if the sequence $\left(p_{i}-p_{i-1}\right)_{i \in \mathbb{Z}}$ is quasi-periodic but not periodic, then we can find a solution $\left(q_{i}\right)_{i \in \mathbb{Z}}$ to the Period Problem such that the associated minimal surface $\mathcal{M}\left[q_{i}: i \in \mathbb{Z}\right]$ is quasi-periodic but not periodic in $\mathbb{R}^{2} \times \mathbb{S}^{1}$.

8.1. Preliminaries. First we need to fix some notation. In the following, an element of $\mathbb{R}^{\mathbb{Z}}$ will be denoted as a function: $x \in \mathbb{R}^{\mathbb{Z}}$ denotes the sequence $(x(i))_{i \in \mathbb{Z} \text {. We }}$ consider on $\mathbb{R}^{\mathbb{Z}}$ the topology of the pointwise convergence, i.e., the sequence $\left(x^{n}\right)_{n \in \mathbb{N}}$ converges to $x^{\infty}$ if for every $i$ : $\lim _{n} x^{n}(i)=x^{\infty}(i)$.

We notice that, for every $A \in \mathbb{R}_{+}$, the subset $[-A, A]^{\mathbb{Z}} \subset \mathbb{R}^{\mathbb{Z}}$ is compact. Besides, on $[-A, A]^{\mathbb{Z}}$, the pointwise convergence is metrizable: if $x, y \in[-A, A]^{\mathbb{Z}}$, we define

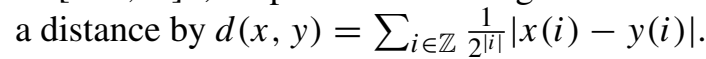

Let $\varphi: \mathbb{N} \rightarrow \mathbb{N}$ be a function. In the following we say that $\varphi$ is an extraction if $\varphi$ is strictly increasing. The group $\mathbb{Z}$ acts on the set $\mathbb{R}^{\mathbb{Z}}$ by shift: if $x \in \mathbb{R}^{\mathbb{Z}}$ and $n \in \mathbb{Z}$, we

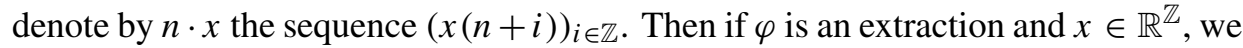
define the sequence $\varphi \cdot x=(\varphi(n) \cdot x)_{n \in \mathbb{N}}$ in $\mathbb{R}^{\mathbb{Z}}$. We have the following definitions.

Definition 2. Let $x$ be in $\mathbb{R}^{\mathbb{Z}}$, this sequence is said to be quasi-periodic if there exists an extraction $\varphi$ such that the sequence $\varphi \cdot x$ converges pointwise to $x$ (namely, for all $\left.i, \lim _{n} x(i+\varphi(n))=x(i)\right)$.

Let $x \in \mathbb{R}^{\mathbb{Z}}$ be an increasing sequence, we say that $x$ has quasi-periodic gaps if the sequence $(x(i)-x(i-1))_{i \in \mathbb{Z}}$ is quasi-periodic.

Let us give two examples:

(1) let $\alpha$ be an irrational number, let $x(i)=[\alpha i]$ be the integer part of $\alpha i$ and let $g(i)=x(i)-x(i-1)$. Then the sequence $(g(i))_{i \in \mathbb{Z}}$ is quasi-periodic and is not periodic. Moreover, for any extraction $\varphi$, if $\lim _{n \rightarrow \infty} \varphi(n) \cdot g$ exists, then it is not periodic.

(2) (the counting sequence) consider the infinite word on the alphabet $\{0, \ldots, 9\}$ formed by writing in order all natural integers:

$$
0123456789101112131415161718192021 \ldots
$$

For $i \geq 1$, let $x(i)$ be the $i$ th digit in this word. For $i \leq 0$, let $x(i)=0$. The sequence $(x(i))_{i \in \mathbb{Z}}$ is quasi-periodic but not periodic. However, if $\varphi$ is an extraction, the limit of $\varphi(n) \cdot x$ can very well be periodic (in fact it can be any sequence of integers between 0 and 9 ). 
8.2. Why are we not yet done? Let us assume that the sequence $(p(i))_{i \in \mathbb{Z}}$ has quasi-periodic gaps, and let $(q(i))_{i \in \mathbb{Z}}$ be a sequence that solves the Period Problem

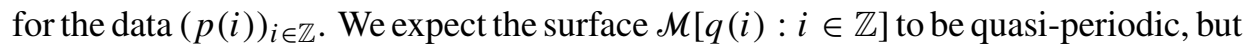
unfortunately we cannot prove that. What we can prove is that there exists a sequence of translations $T_{n}$ such that $T_{n}(\mathcal{M}[q(i): i \in \mathbb{Z}])$ converges to $\mathcal{M}\left[q^{\prime}(i): i \in \mathbb{Z}\right]$, where

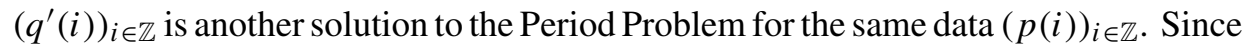
we do not know whether the Period Problem has a unique solution, we cannot ensure that $q^{\prime}(i)=q(i)$. (If the reader knows that the solution to the Period Problem is unique, he may omit what follows. He should also inform the authors).

Our strategy is to prove, using Zorn's lemma, that amongst all the solutions $(q(i))_{i \in \mathbb{Z}}$ to the Period Problem, at least one of them yields a quasi-periodic minimal surface.

8.3. Quasi-periodic surfaces. Let us consider $\ell$ and $\eta_{0}$ as in Proposition 5 .

Let us now explain how we shall construct a quasi-periodic minimal surface. Let $p=(p(i))_{i \in \mathbb{Z}}$ be a strictly increasing sequence with quasi-periodic gaps. In the following, we always assume that $p(0)=0$. The sequence $g=(p(i)-p(i-1))_{i \in \mathbb{Z}}$ is quasi-periodic, we then denote by $\mathcal{A}$ the non-empty set of all extractions $\varphi: \mathbb{N} \rightarrow \mathbb{N}$ such that $\lim _{n \rightarrow \infty} \varphi(n) \cdot g=g$.

Let us fix a sequence $q=(q(i))_{i \in \mathbb{Z}}$ that solves the Period Problem for the data

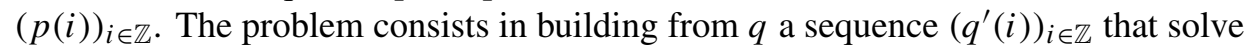
the Period Problem for the data $(p(i))_{i \in \mathbb{Z}}$ and such that $\mathcal{M}\left[q^{\prime}(i): i \in \mathbb{Z}\right]$ is quasiperiodic.

Let us denote by $r$ the sequence $q-2 p: r(i)=q(i)-2 p(i)$ for all $i \in \mathbb{Z}$. Let $\varphi$ be in $\mathcal{A} ; \varphi \cdot r$ is a sequence of elements of $\left[-\eta_{0}, \eta_{0}\right]^{\mathbb{Z}}$. This set is compact so there exists a subsequence of $(\varphi(n) \cdot r)_{n \in \mathbb{N}}$ that converges in $\left[-\eta_{0}, \eta_{0}\right]^{\mathbb{Z}}$. Thus there exists an extraction $\psi: \mathbb{N} \rightarrow \mathbb{N}$ such that $(\varphi \circ \psi) \cdot r$ converges. We notice that, since $\varphi \in \mathcal{A}$, $\varphi \circ \psi \in \mathcal{A}$. The following result describes such a situation.

Proposition 11. With the above notation, let $\varphi \in \mathcal{A}$ such that $\lim _{n \rightarrow \infty} \varphi(n) \cdot r=r^{\prime}$. Then $2 p+r^{\prime}=\left(2 p(i)+r^{\prime}(i)\right)_{i \in \mathbb{Z}}$ solves the Period Problem for the data $(p(i))_{i \in \mathbb{Z}}$.

Proof. For every $n \in \mathbb{N}$, let us define the sequence $q^{n}$ by $q^{n}(i)=q(i+\varphi(n))-$ $2 p(\varphi(n))$ for all $i \in \mathbb{Z}$. We also define $p^{n}$ by $p^{n}(i)=p(i+\varphi(n))-p(\varphi(n))$ for all $i \in \mathbb{Z}$.

Claim 2. We have $\lim p^{n}=p$ and $\lim q^{n}=2 p+r^{\prime}$.

Proof. Let us fix $i \in \mathbb{Z}$ then, if $i \geq 1$ :

$$
p^{n}(i)=p(i+\varphi(n))-p(\varphi(n))=\sum_{l=1+\varphi(n)}^{i+\varphi(n)} p(l)-p(l-1)=
$$




$$
=\sum_{l=1+\varphi(n)}^{i+\varphi(n)} g(l)=\sum_{l=1}^{i} \varphi(n) \cdot g(l)
$$

Since $\lim _{n \rightarrow \infty} \varphi(n) \cdot g=g$, the right-hand term converges to $\sum_{l=1}^{i} g(l)=p(i)$. When $i<1$ we have:

$$
p^{n}(i)=p(i+\varphi(n))-p(\varphi(n))=\sum_{l=i+\varphi(n)+1}^{\varphi(n)} p(l-1)-p(l)=-\sum_{l=i+1}^{0} \varphi(n) \cdot g(l) \text {. }
$$

The right-hand term converges again to $p(i)$. Then $\lim p^{n}=p$.

We have $q^{n}(i)=r(i+\varphi(n))+2 p(i+\varphi(n))-2 p(\varphi(n))=\varphi(n) \cdot r(i)+2 p^{n}(i)$ for all $i \in \mathbb{Z}$. Since $\lim _{n \rightarrow \infty} \varphi(n) \cdot r=r^{\prime}, \lim q^{n}=2 p+r$.

By definition of $q^{n}$, the uniqueness of the solution to the Dirichlet problem implies that we have

$$
v\left[q^{n}(i): i \in \mathbb{Z}\right](x, y)=v[q(i): i \in \mathbb{Z}](x+2 p(\varphi(n)), y) .
$$

This implies that $\left(q^{n}(i)\right)_{i \in \mathbb{Z}}$ solves the Period Problem: $F_{j}\left(q^{n}(i): i \in \mathbb{Z}\right)=0$ for every $j \in \mathbb{Z}$.

Then by Proposition 8 and Claim 2, $2 p+r^{\prime}$ solves the Period Problem for the data $(p(i))_{i \in \mathbb{Z} \text {. }}$

Proposition 6 does not give us the uniqueness of the sequence $(q(i))_{i \in \mathbb{Z}}$ that solves

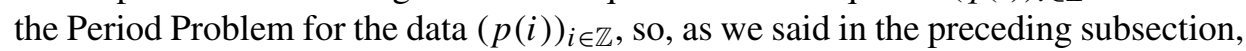
we cannot ensure that the sequences $r$ and $r^{\prime}$ are equal. Such an affirmation would be interesting because of the following proposition.

Proposition 12. With the above notations, if there exists an extraction $\varphi \in \mathcal{A}$ such that $\lim _{n \rightarrow \infty} \varphi(n) \cdot r=r$, the surface $\mathcal{M}[q(i): i \in \mathbb{Z}]$ is quasi-periodic.

Proof. We use the notations of the proof of Proposition 11. We have the sequences $q^{n}, p^{n}$. Now Claim 2 says us that $\lim q^{n}=q$. Let us recall that when $(a(i))_{i \in \mathbb{Z}}$ solves the Period Problem, the surface $\mathcal{M}[a(i): i \in \mathbb{Z}]$ is normalized such that the conjugate to the point in the graph above $(-1,0)$ is the point $(0,0, v(-1,0))$ where $v=v[a(i): i \in \mathbb{Z}]$.

As above, (12) is true. So our normalization for the surfaces $\mathcal{M}$ implies that $\mathcal{M}\left[q^{n}(i): i \in \mathbb{Z}\right]$ is the image of $\mathcal{M}[q(i): i \in \mathbb{Z}]$ by an horizontal translation $T_{n}$. The vector of the translation is $\left(0,-X_{2}^{*}(2 p(\varphi(n))-1)\right)$ where $X_{2}^{*}$ is $X_{2}^{*}[q(i): i \in \mathbb{Z}]$.

Then by Proposition 8 and Claim 2, the sequence of minimal surfaces $\left(\mathcal{M}\left[q^{n}(i)\right.\right.$ : $i \in \mathbb{Z}])_{n \in \mathbb{N}}=\left(T_{n}(\mathcal{M}[q(i): i \in \mathbb{Z}])\right)_{n \in \mathbb{N}}$ converges to $\mathcal{M}[q(i): i \in \mathbb{Z}]$ smoothly on compact subsets of $\mathbb{R}^{2} \times \mathbb{S}^{1}$. Since $\mathcal{M}[q(i): i \in \mathbb{Z}]$ is properly embedded, 
$\lim \left|X_{2}^{*}(2 p(\varphi(n))-1)\right|=\infty$; thus $\left(T_{n}\right)_{n \in \mathbb{N}}$ is a diverging sequence of translations. This proves that $\mathcal{M}[q(i): i \in \mathbb{Z}]$ is quasi-periodic.

By using a proposition that will be proved in the next subsection we then can prove our main theorem.

Theorem 4. Let $(p(i))_{i \in \mathbb{Z}}$ be a sequence with quasi-periodic gaps that satisfies the hypotheses of Proposition 6. Then there exists a sequence $(q(i))_{i \in \mathbb{Z}}$ which solves the Period Problem for the data $(p(i))_{i \in \mathbb{Z}}$ and such that $\mathcal{M}[q(i): i \in \mathbb{Z}]$ is quasiperiodic. Moreover if $(p(i+1)-p(i))_{i \in \mathbb{Z}}$ is not periodic, $\mathcal{M}[q(i): i \in \mathbb{Z}]$ is not periodic.

Proof. By Proposition 6, there exists a sequence $(q(i))_{i \in \mathbb{Z}}$ that solves the Period Problem for the data $(p(i))_{i \in \mathbb{Z} \text {. }}$

The sequence $(g(i))_{i \in \mathbb{Z}}=(p(i)-p(i-1))_{i \in \mathbb{Z}}$ is quasi-periodic so we have the set $\mathcal{A}$. Let $r$ denotes the sequence $q-2 p$, we recall that $r \in\left[-\eta_{0}, \eta_{0}\right]^{\mathbb{Z}}$. By Proposition 13, there exists $\varphi$ and $\psi \in \mathcal{A}$ such that

$$
\begin{aligned}
\lim \varphi \cdot r & =r^{\prime}, \\
\lim \psi \cdot r^{\prime} & =r^{\prime} .
\end{aligned}
$$

By Proposition 11, equation (13) implies that the sequence $2 p+r^{\prime}$ solves the

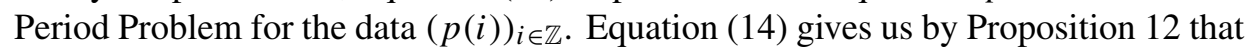
$\mathcal{M}\left[2 p(i)+r^{\prime}(i): i \in \mathbb{Z}\right]$ is quasi-periodic.

8.4. A dynamical result. Let $X$ be a topological space with a countable basis. In the following, we shall denote by $\left(V_{n}(x)\right)_{n \in \mathbb{N}}$ a countable decreasing basis of open neighborhoods of $x \in X$. Let $F: X \rightarrow X$ be a continuous map. Let $g$ be in $X$. We assume that there exists an extraction $\varphi$ such that $\lim _{n} F^{\varphi(n)}(g)=g$. As above we denote by $\mathcal{A}$ the set of extractions $\varphi$ such that $\lim _{n} F^{\varphi(n)}(g)=g$. The aim of this section is to prove the following proposition.

Proposition 13. Let $K$ be a compact subset of $X$ such that $F(K) \subset K$. Let $x$ be in $K$. Then there exists two extractions $\varphi \in \mathcal{A}$ and $\psi \in \mathcal{A}$ such that $\lim _{n} F^{\varphi(n)}(x)=x^{\prime}$ and $\lim _{n} F^{\psi(n)}\left(x^{\prime}\right)=x^{\prime}$.

In the proof of Theorem 4, we use this result with $X=\mathbb{R}^{\mathbb{Z}}$ with its pointwise convergence topology, $K$ is $\left[-\eta_{0}, \eta_{0}\right]^{\mathbb{Z}}, F$ is the shift map and $g$ is the quasi-periodic sequence $g$.

Before proving the above proposition, let us fix some notations. Let $x$ be as in the proposition and $\varphi \in \mathcal{A}$; the sequence $F^{\varphi(n)}(x)$ is a sequence in $K$ which is compact. Thus there exists a subsequence that converges. As said above this implies that there 
exists an extraction $\psi$ such that $F^{\varphi(\psi(n))}(x)$ converges. We notice that $\varphi \circ \psi \in \mathcal{A}$. Hence we define

$$
\operatorname{Asymp}(x)=\left\{y \in K \mid y=\lim _{n} F^{\varphi(n)}(x) \text { for some } \varphi \in \mathcal{A}\right\} .
$$

We know that $\operatorname{Asymp}(x)$ is non-empty. In fact Proposition 13 consists in proving that there exists $x^{\prime} \in \operatorname{Asymp}(x)$ such that $x^{\prime} \in \operatorname{Asymp}\left(x^{\prime}\right)$. Then Proposition 13 is a consequence of the following three lemmas.

Lemma 7. Let $x \in K$. Then $\operatorname{Asymp}(x)$ is a closed subset of $K$.

Proof. Let $\left(y_{k}\right)_{k \in \mathbb{N}}$ be a sequence in $\operatorname{Asymp}(x)$ that converges to $y \in K$. For each $k$, we choose $\varphi_{k} \in \mathcal{A}$ such that $y_{k}=\lim _{n} F^{\varphi_{k}(n)}(x)$. We are going to construct by induction $\psi \in \mathcal{A}$ such that $y=\lim _{n} F^{\psi(n)}(x)$.

Let $n$ be in $\mathbb{N}^{*}$, we assume that $\psi(q)$ is constructed for $q<n$ such that, for every $q<n$,

$$
F^{\psi(q)}(g) \in V_{q}(g) \text { and } \quad F^{\psi(q)}(x) \in V_{q}(y) .
$$

Since $\lim y_{k}=y$, there exists $k_{0}$ such that $y_{k_{0}} \in V_{n}(y)$; hence $V_{n}(y)$ is an open neighborhood of $y_{k_{0}}$. Since $\varphi_{k_{0}} \in \mathcal{A}$, there exists $q_{0}$ such that $\varphi_{k_{0}}\left(q_{0}\right)>\psi(n-1)$, and

$$
F^{\varphi_{k_{0}}\left(q_{0}\right)}(g) \in V_{n}(g) \text { and } F^{\varphi_{k_{0}}\left(q_{0}\right)}(x) \in V_{n}(y) .
$$

Then if we take $\psi(n)=\varphi_{k_{0}}\left(q_{0}\right)$ we get

$$
\begin{aligned}
& F^{\psi(n)}(g) \in V_{n}(g), \\
& F^{\psi(n)}(x) \in V_{n}(y) .
\end{aligned}
$$

This finishes our construction.

Equation (15) implies that $\psi \in \mathcal{A}$ and (16) implies that $\lim _{n} F^{\psi(n)}(x)=y$ thus $y \in \operatorname{Asymp}(x)$.

Lemma 8. Let $x \in K$ and let $y \in \operatorname{Asymp}(x)$. Then $\operatorname{Asymp}(y) \subset \operatorname{Asymp}(x)$.

Proof. Let $z$ be in $\operatorname{Asymp}(y)$. Let $\varphi$ and $\psi \in \mathcal{A}$ such that $\lim _{n} F^{\varphi(n)}(x)=y$ and $\lim _{n} F^{\psi(n)}(y)=z$. Let us build by induction $\chi \in \mathcal{A}$ such that $\lim _{n} F^{\chi(n)}(x)=z$.

Let $n$ be in $\mathbb{N}^{*}$. We assume that $\chi(q)$ is constructed for $q<n$ such that, for every $q<n$,

$$
F^{\chi(q)}(g) \in V_{q}(g) \quad \text { and } \quad F^{\chi(q)}(x) \in V_{q}(z)
$$


Since $\lim _{n} F^{\psi(n)}(y)=z$, there exists $k_{0}$ such that

$$
F^{\psi\left(k_{0}\right)}(g) \in V_{n}(g) \text { and } F^{\psi\left(k_{0}\right)}(y) \in V_{n}(z) .
$$

Then $\left(F^{\psi\left(k_{0}\right)}\right)^{-1}\left(V_{n}(g)\right)$ is an open neighborhood of $g$ and $\left(F^{\psi\left(k_{0}\right)}\right)^{-1}\left(V_{n}(z)\right)$ is an open neighborhood of $y$. Since $\lim _{n} F^{\varphi(n)}(x)=y$, there exists $l_{0}$ such that $\psi\left(k_{0}\right)+\varphi\left(l_{0}\right)>\chi(n-1)$, and

$$
F^{\varphi\left(l_{0}\right)}(g) \in\left(F^{\psi\left(k_{0}\right)}\right)^{-1}\left(V_{n}(g)\right) \quad \text { and } \quad F^{\varphi\left(l_{0}\right)}(x) \in\left(F^{\psi\left(k_{0}\right)}\right)^{-1}\left(V_{n}(z)\right)
$$

Hence if we take $\chi(n)=\psi\left(k_{0}\right)+\varphi\left(l_{0}\right)$, we have:

$$
\begin{aligned}
& F^{\chi(n)}(g) \in V_{n}(g), \\
& F^{\chi(n)}(x) \in V_{n}(z) .
\end{aligned}
$$

This finishes our construction.

Equation (17) implies that $\chi$ is in $\mathcal{A}$ and (18) gives us that $\lim _{n} F^{\chi(n)}(x)=z$, hence $z \in \operatorname{Asymp}(x)$.

Lemma 9. Let $K$ be a compact set and $T: K \rightarrow \mathcal{P}(K)$ a map such that:

(1) for all $x \in K, T(x)$ is closed and non-empty;

(2) for all $x \in K$ and all $y \in T(x), T(y) \subset T(x)$.

Let $x \in K$, then there exists $y \in T(x)$ such that $y \in T(y)$.

Proposition 13 is then a consequence of this lemma with $T=$ Asymp.

Proof of Lemma 9. The proof of this lemma is given by Zorn's lemma. Let $x$ be in $K$, we denote by $\mathcal{B}$ the set $\{T(y), y \in T(x)\}$. $\mathscr{B}$ is ordered by the inclusion. Let $\left(T_{i}\right)_{i \in I}$ be a totally ordered family of $\mathscr{B}$. Let us define $T_{\infty}=\bigcap_{i \in I} T_{i}$. If $T_{\infty}$ is empty, since each $T_{i}$ is closed and $K$ is compact there exists a finite subset $I_{0} \subset I$ such that $\bigcap_{i \in I_{0}} T_{i}=\emptyset$. Since $\left(T_{i}\right)_{i \in I_{0}}$ is totally ordered there exists $i_{0} \in I_{0}$ such that $T_{i_{0}}=\bigcap_{i \in I_{0}} T_{i}$, but $T_{i_{0}}$ is non-empty thus $T_{\infty} \neq \emptyset$.

Let $y$ be in $T_{\infty}$, then $y \in T_{i}$ for all $i \in I$. This implies by the second hypothesis that $y \in T(x)$ and $T(y) \in \mathcal{B}$. Besides $T(y) \subset T_{i}$ for all $i \in I$; then $T(y) \subset T_{\infty}$. We obtain that $T(y)$ is an under-bound for the family $\left(T_{i}\right)_{i \in I}$.

We have proved that every totally ordered family admits an under-bound. Hence, by Zorn's lemma, there exists an element $T_{m} \in \mathscr{B}$ which is minimal for the inclusion. Let $y$ be in $T_{m}$ (we recall that all elements of $\mathscr{B}$ are non-empty subsets of $K$ ). We have $y \in T(x)$ by the second hypothesis then $T(y) \in \mathscr{B}$ and $T(y) \subset T_{m}$. Since $T_{m}$ is minimal in $\mathcal{B}, T(y)=T_{m}$ and $y \in T(y)$. 


\section{References}

[1] J. L. Barbosa and M. do Carmo, On the size of a stable minimal surface in $\mathbb{R}^{3}$. Amer. J. Math. 98 (1976), 515-528. Zbl 0332.53006 MR 0413172

[2] R. Bartnik and L. Simon, Spacelike hypersurfaces with prescribed boundary values and mean curvature. Comm. Math. Phys. 87 (1982/83), 131-152.Zbl 0512.53055 MR 0680653

[3] H. Jenkins and J. Serrin, Variational problems of minimal surface type II. Boundary value problems for the minimal surface equation. Arch. Rational Mech. Anal. 21 (1966), 321-342. Zbl 0171.08301 MR 0190811

[4] H. Karcher, Embedded minimal surfaces derived from Scherk's examples. Manuscripta Math. 62 (1988), 83-114. MR0958255, Zbl 658.53006. Zbl 0658.53006 MR 0958255

[5] A. A. Klyachin and V. M. Miklyukov, Traces of functions with spacelike graphs and a problem of extension with constraints imposed on the gradient. Math. Sb. 183 (1992), 49-64. Zbl 0791.53023 MR 1186974

[6] L. Mazet, M. Rodriguez, and M. Traizet, Saddle towers with infinitely many ends. Indiana Univ. Math. J. 56 (2007), 2821-2838. Zbl 05254001 MR 2375703

[7] L. Mazet, The Dirichlet problem for the minimal surfaces equation and the Plateau problem at infinity. J. Inst. Math. Jussieu 3 (2004), 397-420. Zbl 1063.53007 MR 2074430

[8] L. Mazet, Quelques résultats de non-existence pour l'équation des surfaces minimales. Bull. Sci. Math. 128 (2004), 577-586. Zbl 1082.53010 MR 2081087

[9] L. Mazet, The plateau problem at infinity for horinzontal ends and genus 1. Indiana Univ. Math. J. 55 (2006), 15-64. Zbl 1105.53007 MR 2207546

[10] L. Mazet, A uniqueness result for maximal surfaces in Minkowski 3-space. C. R. Math. Acad. Sci. Paris 344 (2007), 785-790. Zbl 1125.53047 MR 2340448

[11] W. H. Meeks III, J. Pérez, and A. Ros, The geometry of minimal surfaces of finite genus I; curvature estimates and quasiperiodicity. J. Differential Geom. 66 (2004), 1-45. Zbl 1068.53012 MR 2128712

[12] W. H. Meeks III, J. Pérez, and A. Ros, Uniqueness of the Riemann minimal surfaces. In Global theory of minimal surfaces, Clay Math. Proc. 2, Amer. Math. Soc., Providence, RI, 2005, 597-610. Zbl 1115.53009 MR 2167278

[13] W. H. Meeks III, J. Perez, and A. Ros, Embedded minimal surfaces: removable singularities, local pictures and parking garage structures, the dynamics of dilation invariant collections and the characterization of examples of quadratic curvature decay. Preprint, 2006.

[14] W. H. Meeks III and H. Rosenberg, Maximum principles at infinity. Preprint, 2006.

[15] A. Ros, Embedded minimal surfaces: forces, topology and symmetries. Calc. Var. 4(1996), 469-496. MR1402733, Zbl 861.53008. Zbl 0861.53008 MR 1402733

[16] W. Rossman, E. C. Thayer, and M. Wohlgemuth, Doubly periodic minimal surfaces. Experiment. Math. 9 (2000), 197-219. Zbl 1031.53014 MR 1780206

[17] R. Schoen, Estimates for Stable Minimal Surfaces in Three Dimensional Manifolds. Ann. of Math. Stud. 103 Princeton University Press, Princeton, NJ, 1983. Zbl 0532.53042 MR 0795231 
[18] F. Wei, Some existence and uniqueness theorems for doubly periodic minimal surfaces. Invent. Math. 109 (1992), 113-136. Zbl 773.53005 MR 1168368

Received September 21, 2006

Laurent Mazet, Université Paris-Est, Laboratoire d'Analyse et Mathématiques Appliquées, UMR 8050, UFR de Sciences et technologies, 61 avenue du Général de Gaulle,

94010 Créteil cedex, France

E-mail: laurent.mazet@math.cnrs.fr

Martin Traizet, Laboratoire de Mathématiques et Physique Théorique, Faculté des Sciences et Techniques, Université de Tours, Parc de Grandmont, 37200 Tours, France

E-mail: martin.traizet@1mpt.univ-tours.fr 